\title{
Properties of M31
}

\section{Dust. Basic properties and a discussion about age-dependent dust heating}

\author{
M. Montalto ${ }^{1,2}$, S. Seitz ${ }^{1,2}$, A. Riffeser ${ }^{1}$, U. Hopp ${ }^{1,2}$, C.-H. Lee ${ }^{1}$, and R. Schönrich ${ }^{1}$ \\ ${ }^{1}$ University Observatory Munich, Scheinerstrasse 1, 81679 Munich, Germany \\ e-mail: montalto@usm.uni-muenchen.de \\ 2 Max-Planck Institute for Extraterrestrial Physics, Giessenbachstrasse, 85748 Garching, Germany
}

Received 25 March 2009 / Accepted 4 July 2009

\section{ABSTRACT}

\begin{abstract}
Aims. Observations acquired by the Spitzer Space Telescope and improvements to theoretical modeling of dust emission properties are used to discuss the distribution of dust and its characteristics in the closest neighbor spiral galaxy M31. These data are then used with GALEX FUV, NUV, and SDSS images to study the age dependence of the dust heating process.

Methods. Spitzer IRAC/MIPS maps of M31 were matched together and compared to dust emission models allowing us to constrain the dust mass, the intensity of the mean radiation field, the abundance of polycyclic aromatic hydrocarbon (PAH) particles. The total infrared emission (TIR) was analyzed as a function of UV and optical colors and compared to predictions of models that consider age-dependent dust heating.

Results. We demonstrate that cold-dust component emission dominates the infrared spectral energy distribution of M31. The mean intensity of the radiation field heating the dust is low (typically $U<2$, where $U=1$ is the value in the solar neighborhood). Because of a lack of submillimeter observations, the dust mass $\left(M_{\text {dust }}\right)$ is only weakly constrained by the infrared spectrum, but we derived a lower limit of $M_{\text {dust }} \gtrsim 1.1 \times 10^{7} M_{\odot}$ with a best-fit model value of $M_{\text {dust }}=7.6 \times 10^{7} M_{\odot}$, in good agreement with expectations from $\mathrm{CO}$ and $\mathrm{HI}$ measurements. Across the spiral-ring structure of M31, we show that a fraction $>3 \%$ of the total dust mass is in PAHs. UV and optical colors are correlated with the total infrared to far ultraviolet (TIR/FUV) ratios in $\sim 670$ pc-sized regions over the disk of M31, although deviating from the relationship between infrared excess and ultraviolet spectral slope (referred as IRX- $\beta$ relationship) for starburst galaxies. In particular, redder regions have lower values of the TIR/FUV ratio for a fixed color. Considering the predictions of models that account for the dust-heating age dependence, we found that in $83 \%$ of the regions analyzed across the $10 \mathrm{kpc}$ ring, more than $50 \%$ of the energy absorbed by the dust is rediated at $\lambda>4000 \AA$ and that dust in M31 appears mainly heated by populations a few Gyr old even across the star-forming ring. We also found that the attenuation varies radially peaking close to $10 \mathrm{kpc}$ and decreases more rapidly with radius in the inner regions of M31 than in the outer regions in agreement with previous studies. We finally derived the attenuation map of M31 at $6^{\prime \prime} / \mathrm{px}$ resolution ( 100 pc/px along the plane of M31).
\end{abstract}

Conclusions.

Key words. dust, extinction

\section{Introduction}

The presence of the dust affects astrophysical observations in different ways. In the UV-optical spectral region, the dust absorbs the stellar radiation field, while it re-emits the absorbed energy in the IR-FIR and sub-millimetric spectral regions. It is thus essential to determine the dust properties a given system as accurately as possible. Although in our own Galaxy we can complete observations of very high spatial resolutions and accuracies, our particular location inside the galaxy restricts the objectivity of our analysis.

Being the closest large spiral similar to the Milky Way, M31 offers a fascinating view of the dust distribution across the disk of a galaxy and a unique opportunity to study the properties of a major disk galaxy in detail. Previous studies focused on the dust in this galaxy, but new observational results and theoretical improvements now allow some issues to be revisited and

\footnotetext{
* Maps of Figure 14 and the $E(B-V)$ map of Figure 17 are only available in electronic form at the CDS via anonymous ftp to cdsarc.u-strasbg.fr (130.79.128.5) or via http://cdsweb.u-strasbg.fr/cgi-bin/qcat?J/A+A/507/283
}

critically discussed and allow us to shed new light on unresolved problems. In particular, GALEX UV observations (Gil de Paz et al. 2007), SDSS optical images (York et al. 2000), and Spitzer Space Telescope observations (Barmby et al. 2006; Gordon et al. 2006) in the infrared and far infrared are now available for M31. Together with recent CO and HI observations (Nieten et al. 2006; Braun et al. 2009), this new wealth of data allows a much more detailed exploration of the dust properties than previous studies. Spitzer data allow us to study the far-infrared emission on significantly smaller scales than in the IRAS era.

Important progress has also been made in the theoretical understanding of dust properties, which has enabled new parameters to be derived from the data (Weingartner \& Draine 2001; Draine \& Li 2007; Draine et al. 2007). This work is thus devoted to an analysis of data presently available for M31 using examples of the latest theoretical models. We focus on the mean radiation field heating the dust, on the dust mass determination, and on the amount and distribution of polycyclic aromatic hydocarbon particles (PAHs) in M31.

M31 is an invaluable target for studying a problem that has attracted a great deal of attention in recent studies: what is the 
main heating source of dust in a galaxy? The dust certainly tends to preferentially absorb UV photons, so naively one would of course expect UV radiation, especially from the young populations detected in the GALEX images of M31, to be the main source responsible for the observed dust emission. This idea clearly holds for starburst and very actively star-forming galaxies (see e.g., Buat 1992; Meurer et al. 1995) and led to the discovery of the IRX- $\beta$ relation (Meurer et al. 1999). However, in galaxies hosting older stellar populations, the bulk of the radiation is emitted at longer wavelengths. Studies have demonstrated that in systems with relatively low star-formation rates, old stars can contribute significantly to the dust heating (Cortese et al. 2008; Kong et al. 2004; Buat et al. 2005; Gordon et al. 2000), and we demonstrate in this paper that the latter also applies to M31. This difference is crucial when one attempts to derive accurate dust attenutations and, consequently, star-formation rates in any galactic system.

The structure of this paper is as follows: In Sect. 2, we present the analyzed data and the calibration procedure. In Sect. 3, we describe the dust models and their application to determine the dust mass, the mean intensity of the radiation field responsible for dust heating, and the PAHs abundances in M31. In Sect. 4, we discuss the age dependency of dust heating, deriving the $A_{\mathrm{FUV}}$ attenuation across the spiral-ring structure of M31. Finally, in Sects. 5 and 6 we discuss and summarize our results.

\section{The data}

\subsection{IR}

In this work we used the IR images of M31 described in Barmby et al. (2006) and Gordon et al. (2006), obtained with the IRAC/MIPS instruments onboard of the Spitzer Space Telescope (SST). In particular, we retrieved from the SST archive the $3.6 \mu \mathrm{m}, 8 \mu \mathrm{m}$ IRAC, and the $24 \mu \mathrm{m}, 70 \mu \mathrm{m}$ and $160 \mu \mathrm{m}$ MIPS Basic Calibrated Data (BCDs) processed by the SST's archive pipeline (v.14). The BCDs were then stacked together using the MOPEX software (version 18.1).

For IRAC observations, we applied an overlap correction before mosaiking to match the background between adjacent images, and discarded the first frame of each observing run because it was typically acquired with a lower bias level than subsequent images (first-frame effect). The background was evaluated in the external regions and subtracted from the images with the IRAF imsurfit task using a low-order polynomial (first or second order depending on the map). We then applied the IRAC photometric corrections to infinite sources as reported in the IRAC documentation multiplying the $3.6 \mu \mathrm{m}$ image by 0.91 and the $8 \mu \mathrm{m}$ image by 0.74 to account for the scattering of incident light in the array focal planes. We applied the correction to extended sources because in this work we focus on the diffuse dust emission, not on point-like sources.

For MIPS $70 \mu \mathrm{m}$ and $160 \mu \mathrm{m}$ data, we used our customized software for background subtraction by means of fitting and subtracting a first order polynomial along the scan direction excluding the M31 region. The final MIPS mosaic images have a resolution of $6^{\prime \prime} / \mathrm{px}, 18^{\prime \prime} / \mathrm{px}$, and $40^{\prime \prime} / \mathrm{px}$ at $24 \mu \mathrm{m}, 70 \mu \mathrm{m}$ and $160 \mu \mathrm{m}$, respectively.

Because our purpose was to directly compare the fluxes in each band on a pixel-by-pixel basis we had to resample the images to the same astrometric reference system. We created two sets of images: in the first one, the IRAC $3.6 \mu \mathrm{m}$ and $8 \mu \mathrm{m}$ images were placed on the reference system of the $24 \mu \mathrm{m}$ mosaic $\left(6^{\prime \prime} / \mathrm{px}\right)$, and in the second, the IRAC $3.6 \mu \mathrm{m}$ and $8 \mu \mathrm{m}$ and the
Table 1. Percentual variation in the total integrated flux of M31 due to the resampling procedure.

\begin{tabular}{ccc}
\hline \hline Band $(\mu \mathrm{m})$ & Resampled to $24 \mu \mathrm{m}(\%)$ & Resampled to $160 \mu \mathrm{m}(\%)$ \\
\hline 3.6 & 2 & 2 \\
7.9 & 3 & 2 \\
24 & - & 0.8 \\
70 & - & 0.6 \\
160 & - & - \\
\hline
\end{tabular}

MIPS $24 \mu \mathrm{m}$ and $70 \mu \mathrm{m}$ images were placed on the reference system of the $160 \mu \mathrm{m}$ mosaic $\left(40^{\prime \prime} / \mathrm{px}\right)$. These two sets will be considered separately in the following. To ensure that the different instrument images are comparable, we first matched the PSF of the input instrument to that of the target instrument (MIPS $24 \mu \mathrm{m}$ or MIPS $160 \mu \mathrm{m})$. This was achieved with the help of the transformation kernels provided by Gordon ${ }^{1}$ (Gordon et al. 2008). These kernels provide the transformation between the PSFs of each couple of IRAC-IRAC, IRAC-MIPS, and MIPSMIPS instruments dependent on the dust temperature. After the convolution, we resampled the images on to the same astrometric grid, in such a way that each pixel in a given band corresponded to the same physical region of the same pixel in the other bands. We estimated the uncertainty in the resampling step by means of a comparison of the integrated photometry on the entire galaxy before and after this procedure, as shown in Table 1 where: the second column presents the percentual variation for the images resampled to the $24 \mu \mathrm{m}$ resolution, the third column for images resampled to $160 \mu \mathrm{m}$. The total integrated flux was measured in the same area in all the images, which corresponds to an ellipse centered on M31, with a semi-major axis of $84^{\prime}$, a semi-minor axis of $18^{\prime}$, and a position angle of $\mathrm{PA}=38^{\circ}$. We found that in the worst case the relative variation in flux was $3 \%$. The uncertainty in the background subtraction was estimated by evaluating the scatter in the background subtracted mosaics in the outermost regions, and found to be negligible.

While creating the mosaics with MOPEX ${ }^{2}$ (MOsaicker and Point source EXtractor), we also derived the associated error map, which provides the error related to the entire reduction procedure performed by the pipeline. To account for other sources of error (resampling, background subtraction, and BCDs calibration) we simply added a constant term equal to $10 \%$, with the exception the $24 \mu \mathrm{m}$ map for which we would have obtained unrealistically large values of the error. In reality, our approach can be considered rather conservative, because as stated in the SST documentation, the standard deviation maps account for the error of the pipeline in a conservative way. Table 2 presents infrared integrated flux measurements of M31 obtained by IRAC (Barmby et al. 2006), MIPS (Gordon et al. 2006), COBE (Odenwald et al. 1998), IRAS (Rice et al. 1988), MSX (Kraemer et al. 2002), and ISO (Haas et al. 1998) instruments. The fourth columns shows our own measurements derived from IRAC and MIPS observations. We measured the integrated flux in an ellipse of semi-major axis $84^{\prime}$ and semi-minor axis $18^{\prime}$, centered on $\mathrm{M} 31$ with a position angle of $\mathrm{PA}=38^{\circ}$. In particular, with respect to the measurements quoted by Barmby et al. (2006) and Gordon et al. (2006), our integrated fluxes are $0.6 \sigma$ smaller, $0.1 \sigma$ smaller, $1 \sigma$ larger, $0.8 \sigma$ larger, and $0.4 \sigma$ smaller than their own measurements at $3.6 \mu \mathrm{m}, 8 \mu \mathrm{m}, 24 \mu \mathrm{m}, 70 \mu \mathrm{m}$, and $160 \mu \mathrm{m}$ respectively, and our estimated error is $9 \%$ smaller, and $30 \%$,

\footnotetext{
1 http://dirty.as.arizona.edu/ kgordon/mips/conv_psfs/ conv_psfp.html

2 http://ssc.spitzer. caltech.edu/postbcd/mopex.html
} 
Table 2. Measurements of M31 infrared integrated fluxes.

\begin{tabular}{|c|c|c|c|c|c|c|c|c|c|}
\hline $\begin{array}{l}\lambda^{a} \\
\mu \mathrm{m}\end{array}$ & $\begin{array}{l}\lambda_{\min }^{a} \\
\mu \mathrm{m}\end{array}$ & $\begin{array}{l}\lambda_{\max }^{a} \\
\mu \mathrm{m}\end{array}$ & $\begin{array}{c}\text { This work } \\
\text { Jy }\end{array}$ & $\begin{array}{c}\text { IRAC } \\
\text { Jy }\end{array}$ & $\begin{array}{l}\text { MIPS } \\
\text { Jy }\end{array}$ & $\begin{array}{c}\text { COBE } \\
\text { Jy }\end{array}$ & $\begin{array}{c}\text { IRAS } \\
\text { Jy }\end{array}$ & $\begin{array}{c}\mathrm{MSX} \\
\mathrm{Jy}\end{array}$ & $\begin{array}{l}\text { ISO } \\
\text { Jy }\end{array}$ \\
\hline 1.27 & 1.1 & 1.5 & & & & $534 \pm 107$ & & & \\
\hline 2.22 & 2.0 & 2.4 & & & & $461 \pm 92$ & & & \\
\hline 3.53 & 3.0 & 4.1 & & & & $245 \pm 49$ & & & \\
\hline 3.55 & 3.2 & 3.9 & $239 \pm 29$ & $259 \pm 32^{b}$ & & & & & \\
\hline 4.88 & 4.5 & 5.3 & & & & $128 \pm 26$ & & & \\
\hline 4.49 & 4.0 & 5.0 & & $144 \pm 20$ & & & & & \\
\hline 5.73 & 5.0 & 6.5 & & $190 \pm 35$ & & & & & \\
\hline 7.87 & 6.4 & 9.5 & $149 \pm 27$ & $151 \pm 21$ & & & & & \\
\hline 8.3 & 6.0 & 10.9 & & & & & & $159 \pm 32$ & \\
\hline 12.0 & 7.6 & 15.4 & & & & & $163 \pm 24$ & & \\
\hline 23.7 & 20.5 & 28.5 & $118 \pm 17$ & & $107 \pm 11$ & & & & \\
\hline 25.0 & 16.5 & 30.4 & & & & & $108 \pm 16$ & & \\
\hline 56.0 & 38.6 & 76.5 & & & & $700 \pm 140$ & & & \\
\hline 60.0 & 37.0 & 82.8 & & & & & $536 \pm 80$ & & \\
\hline 71.4 & 55.1 & 91.6 & $1086 \pm 256$ & & $940 \pm 188$ & & & & \\
\hline 97.7 & 68.6 & 121.8 & & & & $3706 \pm 741$ & & & \\
\hline 100.0 & 74.4 & 130.6 & & & & & $2928 \pm 439$ & & \\
\hline 155.9 & 128.9 & 184.0 & $7315 \pm 1632$ & & $7900 \pm 1580$ & & & & \\
\hline 147.9 & 108.2 & 181.7 & & & & $7545 \pm 1509$ & & & \\
\hline 175.0 & 140.0 & 220.0 & & & & & & & $7900 \pm 800$ \\
\hline 247.9 & 174.7 & 335.9 & & & & $6242 \pm 1248$ & & & \\
\hline
\end{tabular}

${ }^{a}$ The wavelengths reported in the first column are the nominal wavelengths of each instrument waveband. The minimum and maximum wavelengths reported in the second and third columns correspond to a system response (filter+detector) equal to $10 \%$ of the maximum. In particular, the system response functions were taken for Spitzer/IRAC rom http://ssc.spitzer.caltech.edu/irac/spectral_response. html, for Spitzer/MIPS from http://ssc.spitzer.caltech.edu/mips/MIPSfiltsumm.txt, for COBE/DIRBE from http://lambda. gsfc.nasa.gov/data/cobe/dirbe/ancil/spec_resp/DIRBE_SYSTEM_SPECTRAL_RESPONSE_TABLE.ASC, for IRAS from http://irsa . ipac.caltech.edu/IRASdocs/exp.sup/ch2/tabC5.html, for MSX/SpiritIII from http://irsa.ipac.caltech.edu/data/MSX/docs/ MSX_psc_es.pdf (Appendix A, Table A-3), for ISO/ISOPHOT from http://www.mpia.de/ISO/welcome.html; ${ }^{b}$ uncertainties in this table have been taken directly from the literature when available. For COBE measurements, we assumed an error equal to $20 \%$ of the measured flux in each band as suggested by Odenwald et al. (1998) and for IRAS measurements equal to 15\% as suggested by Rice et al. (1988). For our own measurements, the uncertainties have been derived as detailed in the text.

$54 \%, 36 \%$ and $50 \%$ larger, respectively, than their given uncertainties. We conclude that our measurements are consistent within $1 \sigma$ and our errors are in general equivalent to or larger than the values derived by Barmby et al. (2006) and Gordon et al. (2006).

We finally subtracted from the $8 \mu \mathrm{m}$ and the $24 \mu \mathrm{m}$ maps the stellar continuum, which was assumed to be described by the $3.6 \mu \mathrm{m}$ map following Helou et al. (2004):

$F_{v}^{\mathrm{ns}}(8 \mu \mathrm{m})=F_{v}(8 \mu \mathrm{m})-0.232 F_{v}(3.6 \mu \mathrm{m})$
$F_{v}^{\mathrm{ns}}(24 \mu \mathrm{m})=F_{v}(24 \mu \mathrm{m})-0.032 F_{v}(3.6 \mu \mathrm{m})$.

\subsection{Ultraviolet}

The UV maps of M31 were retrieved directly from the NASA Extragalactic Database (NED) and were obtained as part of the survey of nearby galaxies performed with the GALaxy Evolution eXplorer (GALEX) satellite (Gil de Paz et al. 2007). We retrieved and used both the far ultraviolet (FUV) and the near ultraviolet (NUV) images. These maps have a pixel scale of $1.5^{\prime \prime} / \mathrm{px}$, a total integration time of $800 \mathrm{~s}$, and are given in units of counts per pixel per second (CPS). The background in these images can be assumed to be constant and was accurately measured by Gil de Paz et al. (2007), who inferred a mean value of $(9.29 \pm 5.24) \times 10^{-4} \mathrm{CPS}$ and $\left.4.712 \pm 1.529\right) \times 10^{-3} \mathrm{CPS}$ for the FUV and NUV, respectively. We subtracted the background from the maps and then converted the CPS units in Jy by multiplying the factors $f_{0, \mathrm{FUV}}=108 \mu \mathrm{Jy} / \mathrm{CPS}$ and $f_{0, \mathrm{NUV}}=36 \mu \mathrm{Jy} / \mathrm{CPS}$, as given by the GALEX documentation. We then accounted for the foreground Galactic extinction by assuming $E(B-V)=0.062$ in the direction of M31 as reported by Schlegel et al. (1998) with $A_{\mathrm{FUV}}=7.9 E(B-V)$ and $A_{\mathrm{NUV}}=8.0 E(B-V)$ as given by Gil de Paz et al. (2007). Two series of maps were finally derived to compare UV observations with MIPS $24 \mu \mathrm{m}$ and MIPS $160 \mu \mathrm{m}$ data. Both the FUV and the NUV maps were convolved to the corresponding MIPS PSFs and resampled onto pixel grids of $6^{\prime \prime} / \mathrm{px}$ and $40^{\prime \prime} / \mathrm{px}$. The global uncertainty of the final maps was estimated to be $\sim 10 \%$ in flux for both maps.

\subsection{Optical}

We retrieved and stacked together the SDSS images of M31 obtained in the SDSS survey. In this work, we used only the SDSS $i$ band (hereafter $i_{\text {SDSS }}$ ) images of M31. First, we subtracted the SOFTBIAS in every single frame. SOFTBIAS was originally added automatically by the SDSS pipeline to avoid negative pixel values. We aligned each single frame to a large reference image $(1.5 \times 4.2$ square degree $)$ using the IRAF task wregister. We then reconstructed each stripe. The final mosaic image contains 7 stripes (3366-2, 3367-3, 3366-3, 3367-4, 33664, 3367-5, and 3366-5, from left to right in the mosaic image). We first tested for photometric variation, but detented no zeropoint difference between the stripes. Nevertheless, we found sky variations in $y$-direction in different stripes when combining them into the mosaic image. Therefore, we applied an alignment in the $y$-direction for each stripe. Using our customized written software (mupipe/skycalc), we calculated the gradient $a \times y$ and the offset $b$ in flux of the overlapping areas between two 


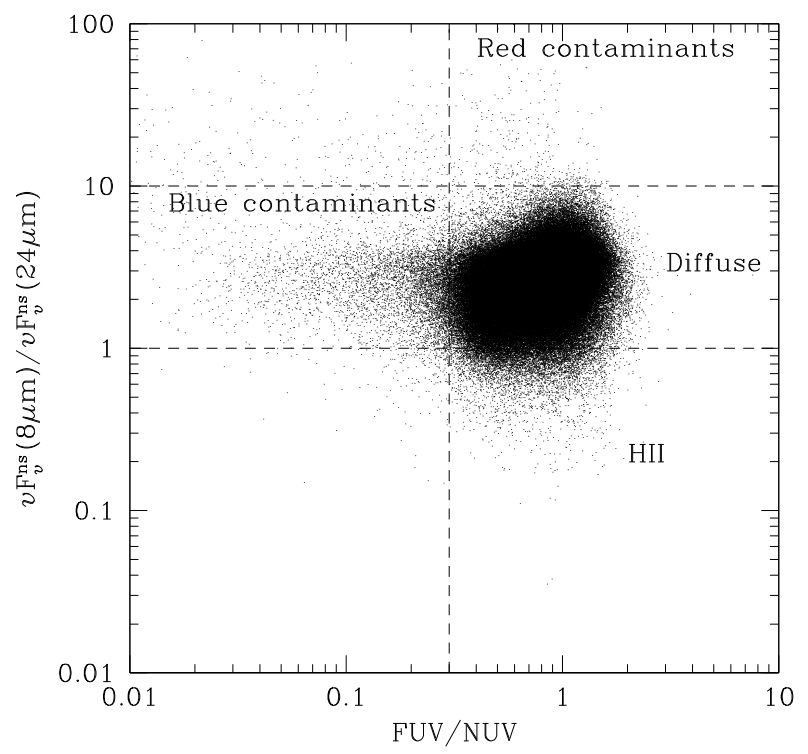

Fig. 1. Two color diagram used to isolate the regions with dust diffuse emission. Labels and dashed lines indicate different sources of emission in function of the colors. $S / N>1$ in all maps and each region corresponds to $6^{\prime \prime} / \mathrm{px}$ resolution.

adjacent stripes, e.g., $F_{3366-2}=F_{3367-3}+a_{3367-3} \times y+b_{3367-3}$ and applied the y-dependent sky correction to the entire stripe. The map was convolved with the Spitzer $160 \mu \mathrm{m}$ PSF and resampled to a $40^{\prime \prime} / \mathrm{px}$ resolution.

We then calibrated the images using catalogued surrounding stars with SDSS photometry and masked out all the sources with $i<17 \mathrm{mag}$, since most of the point-like sources in this magnitude range are foreground contaminants and, excluding the brightest M31 objects (e.g., globular clusters, red supergiants), does not affect our analysis, which is focused on regions dominated by the dust diffuse emission.

To select an uncontaminated sample of regions from the infrared and UV maps, we used the two-color diagram shown in Fig. 1. We corrected the $8 \mu \mathrm{m}$ and $24 \mu \mathrm{m}$ maps for the stellar continuum using Eqs. (1) and (2) and analyzed our data at $6 " / \mathrm{px}$ resolution. We limited the anaysis to regions with $S / N>1$ by excluding from the analysis the inner bulge region $\left(<3^{\prime}\right.$ from the center of M31), partially the interarm regions between the inner spiral-arm and the outermost regions of the galaxy. While the bulk of the data points have colors with $1<v F_{v}^{\mathrm{ns}}(8 \mu \mathrm{m}) / v F_{v}^{\mathrm{ns}}(24 \mu \mathrm{m})<10$ and $0.3<\mathrm{FUV} / N U V<2$, and appear after visual inspection to be associated with the diffuse dust emission, some deviant points are visible in Fig. 1. In our maps, bright point-like sources in the $24 \mu \mathrm{m}$ map have $v F_{v}^{\mathrm{ns}}(8 \mu \mathrm{m}) / v F_{v}^{\mathrm{ns}}(24 \mu \mathrm{m})<1$, and are thus lower than the ratios found in the diffuse component of the dust. Different studies have demonstrated that the emission from point-like sources at $24 \mu \mathrm{m}$ is associated with emission from HII regions in the UV and optical spectral regions (Calzetti et al. 2007; Prescott et al. 2007). The PAHs emission at $7.7 \mu \mathrm{m}$ is also expected to be significantly lower than the diffuse dust emission in these environments (e.g., Calzetti et al. 2005; Thilker et al. 2007; Bendo et al. 2008). On the other hand, regions with $v F_{v}^{\mathrm{ns}}(8 \mu \mathrm{m}) / v F_{v}^{\mathrm{ns}}(24 \mu \mathrm{m})>10$ or $\mathrm{FUV} / N U V<0.3$ appear to be associated with bright foreground stars. We thus adopt the selection criteria for regions: $1<v F_{v}^{\mathrm{ns}}(8 \mu \mathrm{m}) / v F_{v}^{\mathrm{ns}}(24 \mu \mathrm{m})<10$ and FUV $/ N U V>0.3$. In addition to using the SDSS optical map, we added the selection for excluding bright foreground stars as described above.

In Fig. 2, we show for each of the analyzed regions of M31 the minimum signal-to-noise ratio $(S / N)$ when considering all the above-mentioned observational maps together. The $S / N$ is in general higher than 3 along the $10 \mathrm{kpc}$ ring and partially inside the the inner spiral structure. In the interarm and external region, the $S / N$ drops below 1.5. In the bulge region, the $S / N$ is low because of the low stellar-corrected flux at $8 \mu \mathrm{m}$.

\section{The theoretical models}

We used and tested the theoretical models of infrared emission of dust grains heated by starlight developed by Weingartner \& Draine (2001) and Li \& Draine (2001) and updated by Draine \& Li (2007). In the following, we summarize the main model characteristics and results. Dust grains are treated as a mixture of amorphous silicate and carbonaceous grains, where the smallest carbonaceous grains have the physical properties of PAHs. Temperature distribution functions for all particles are calculated and emission spectra provided for dust heated by a single radiation field intensity. The spectral energy distribution of the dust is given by Mathis et al. (1983) (MMP83) and scaled by the dimensionless factor $U$, where $U=1$ corresponds to the mean intensity of the radiation field in the local ISM of the solar neighborhood. We tested the seven Milky Way dust models proposed by Weingartner \& Draine (2001), which have PAH abundances of $0.47 \%, 1.12 \%, 1.77 \%, 2.50 \%, 3.19 \%, 3.90 \%$, and $4.58 \%$ the total dust mass. These models assume a dust-to-gas ratio $M_{\text {dust }} / M_{\mathrm{H}} \simeq 0.01$. For further details, the reader is referred to the studies mentioned above. The emission spectrum predicted by the model can be approximated as

$$
\begin{aligned}
& F_{v \text {,model }}=\Omega_{\text {star }} B_{v}\left(T_{\text {star }}\right) \\
& +\frac{M_{\mathrm{H}}}{4 \pi D^{2}}\left[(1-\gamma) j_{v}^{(0)}\left(\text { model }, U_{\min }\right)+\gamma j_{v}\left(\operatorname{model}, U_{\min }, U_{\max }\right)\right],
\end{aligned}
$$

where the first term accounts for the residual infrared emission originating in the stars and is given by the product of $\Omega_{\text {star }}$ (the solid angle subtended by the stars) and $B_{v}\left(T_{\text {star }}\right)$, the blackbody emissivity with a fixed temperature $T_{\text {star }}=5000 \mathrm{~K}$, which was found by Smith et al. (2007) to provide a good description of the stellar continuum for $\lambda>5 \mu \mathrm{m}$. The distance $D$ of M31 was assumed to be $778 \mathrm{kpc}$, and $j_{v}^{(0)}\left(\operatorname{model}, U_{\min }\right)$ is the dust power radiated per unit frequency per $\mathrm{H}$ nucleon when the dust is exposed to a single radiation field of intensity $U_{\min }$. The factor $\gamma$ $(0 \leq \gamma \leq 1)$ is introduced to parametrize the dust heating effects of a power-law distribution of starlight intensities, as described in Draine \& Li (2007). The calculation of $j_{v}\left(\right.$ model, $\left.U_{\min }, U_{\max }\right)$ infers the dust power radiated per $\mathrm{H}$ nucleon by dust exposed to a power law distribution of starlight intensities $\left(\propto U^{-2}\right)$ corresponding to values of $U$ in the range $U_{\min } \leq U \leq U_{\max }$. The fraction $\gamma(0 \leq \gamma \leq 1)$ of dust emission associated with this intensity field represents dust emission close to $\mathrm{OB}$ associations and/or photodissociation regions, where the intensity $U>U_{\min }$; in this way, this approach allows us to handle dust temperature variations, in a smooth and convenient way. Following Draine et al. (2007), we fixed in our calculation $U_{\max }=10^{6}$. Finally, $M_{\mathrm{H}}$ is the total mass of hydrogen (provided that the models infer the emission per H nucleon). The mass of the dust, $M_{\text {dust }}$, can be obtained from $M_{\text {dust }}=M_{\mathrm{H}}\left(\frac{M_{\text {dust }}}{M_{\mathrm{H}}}\right)$, assuming the dust-to-gas ratio of the models.

Each spectrum is thus completely characterized by 5 free parameters: the solid angle subtended by the stars $\Omega_{\text {star }}$, the kind of emission model (amount of PAHs), the minimum intensity $U_{\min }$ 


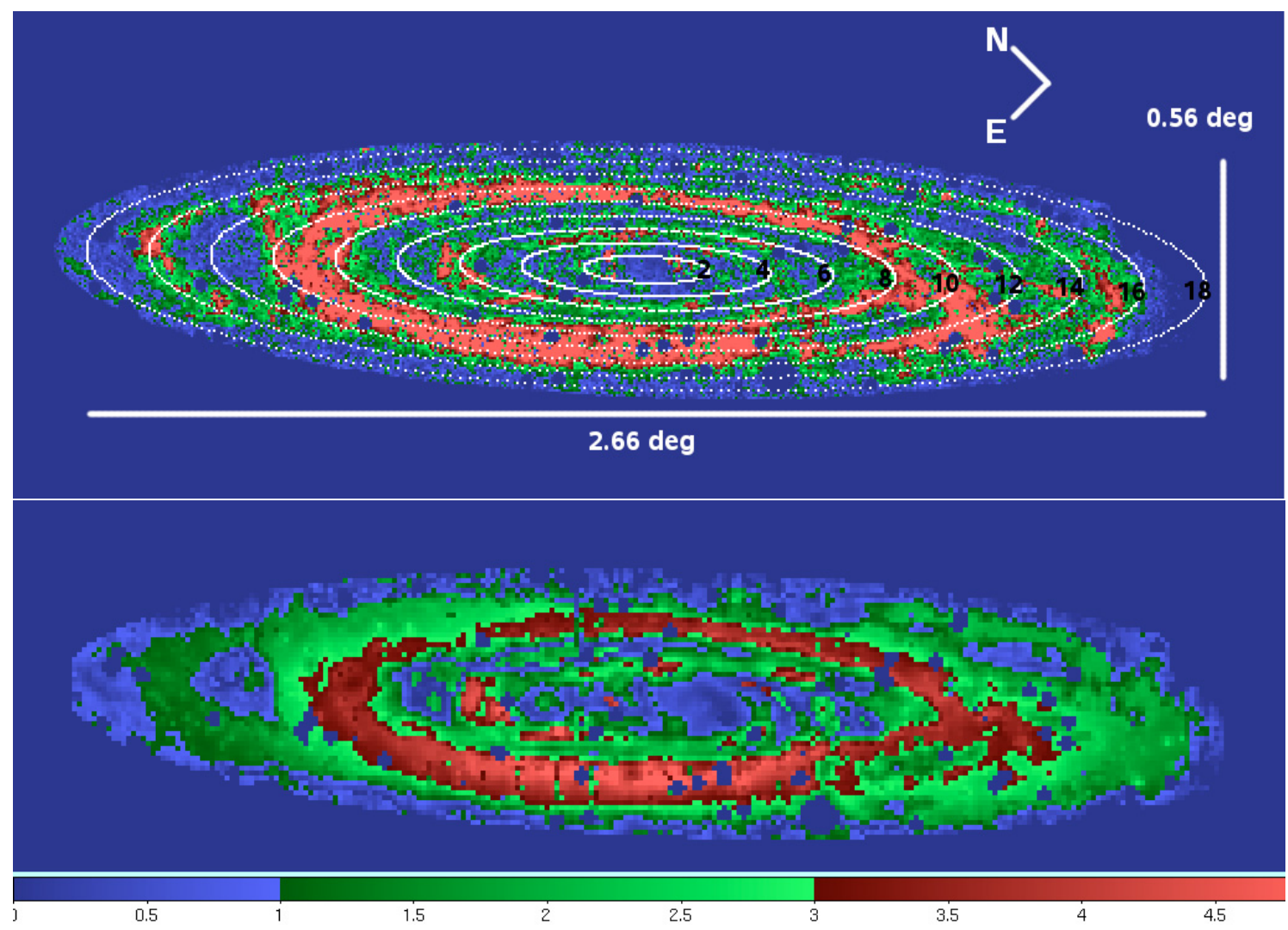

Fig. 2. Upper panel: minimum $S / N$ in each one of the analyzed regions of M31 once considering the FUV, NUV, isDSs, $3.6 \mu \mathrm{m}, 8 \mu \mathrm{m}, 24 \mu \mathrm{m}$, $70 \mu \mathrm{m}$ and $160 \mu \mathrm{m}$ observational maps described in Sect. 2 all together. The resolution is $6 "$ /px. We show also the nine concentric $2 \mathrm{kpc}-$ wide regions used in Sect. 4. Lower panel: the same as the upper panel but for the $40^{\prime \prime} / \mathrm{px}$ resolution maps.

of the starlight radiation field, the fraction $\gamma$ of the total dust mass heated by the power-law distribution of starlight intensities, and the total dust (hydrogen) mass $M_{\mathrm{H}}$. The best-fit model is obtained by minimizing the quantity:

$\chi^{2}=\frac{1}{N_{b}} \sum_{b}^{N_{b}} \frac{\left(F_{v, \text { obs }, b}-\left\langle F_{v, \text { model }}\right\rangle\right)^{2}}{\sigma_{\text {obs }, b}^{2}+\sigma_{\text {model }}^{2}}$.

In Table 2, we collected all the infrared integrated flux measurements of M31 performed to date, by analyzing data obtained with different instruments. In the fourth column, we show our own measurements derived from IRAC and MIPS observations. In general, all these measurements are in good agreement, and define the spectral energy distribution of M31 from $\sim 1 \mu \mathrm{m}$ up to $\sim 250 \mu \mathrm{m}$, as shown in Fig. 3 .

The best-fit model parameters we obtained were: PAHs = $4.6 \%, U_{\min }=0.4, \gamma=0$, and $M_{\text {dust }}=7.6 \times 10^{7} M_{\odot}, \Omega_{\text {star }}=$ $3.1 \times 10^{-16} \mathrm{sr}$, and the $\chi^{2}$ of the best model was $\chi^{2}=0.55$.

In the following, we discuss the uncertainties in the derived parameters and the implications of the results.

\subsection{The uncertainty in the dust mass estimate}

The mass estimate obtained in the above paragraph must be considered to be rather uncertain. This is because, as shown in Fig. 3, the peak wavelength of M31's IR spectrum is close to $160 \mu \mathrm{m}$. Thus, the vast majority of our analyzed observations correspond to shorter waveleghts, therefore colder mass components cannot be reliably constrained by the present observations. The only measurement that provides any form of constraint is the COBE $248 \mu \mathrm{m}$ observation. Otherwise, the cold-mass estimate critically depends on the temperature of the dust inferred from the infrared spectrum. For a given mean radiation field intensity $U$ to which the dust is exposed, the steady-state temperature of the dust is $T \propto U^{1 / 6}$, and the emission peak $\lambda \propto U^{-1 / 6}$ (for grains of dimension $>30 \AA$, Draine \& Li 2007), that is $\mathrm{d} T / T \propto 1 / 6 \mathrm{~d} U / U$. By energy-balance considerations, $U$ is then directly proportional to the emissivity per $\mathrm{H}$ nucleon of the dust grains tabulated in the models, and consequently the dust mass $M_{\text {dust }}$ derived by comparing the model with the observations is $M_{\text {dust }} \propto U^{-1}$, or $\mathrm{d} M_{\text {dust }} / M_{\text {dust }} \propto-\mathrm{d} U / U$. This ensures that the dust temperature estimate is relatively robust to uncertainties in $U$, i.e., a variation of $100 \%$ in $U$ (and in $M_{\text {dust }}$ ) produces a variation of only $\sim 17 \%$ in both dust temperature and emission peak wavelength, to which our measurements are sensitive. Moreover, $\mathrm{d} U / U$ can be quite large even for small changes in $U$, if $U$ is also small, as seems to be the case for M31. To perform a more quantitative analysis, in Fig. 4, we show the $\chi^{2}$ of the best-fit model, obtained fixing the value of $U_{\mathrm{min}}$, for the corresponding total hydrogen mass $M_{\mathrm{H}}$ predicted by the model. The black points (continuous line) in that figure show the result of the fit when all measurements in Table 2 are considered (with the exclusion of ISO and MSX measurements that were not used here), the red points (dotted line) when neglecting the COBE $248 \mu \mathrm{m}$, and the blue (dashed line) points when neglecting the MIPS $160 \mu \mathrm{m}$ and the COBE $148 \mu \mathrm{m}$ and $248 \mu \mathrm{m}$ measurements. From this figure, it is clear that the cold mass cannot be accurately estimated. All the curves exhibit a shallow minimum in $\chi^{2}$ but the range of mass values at which $\chi^{2}$ approaches an absolute minimum is large even when fitting all the present observations. Moreover, when FIR measurements are excluded the $\chi^{2}$ curves are even flatter 


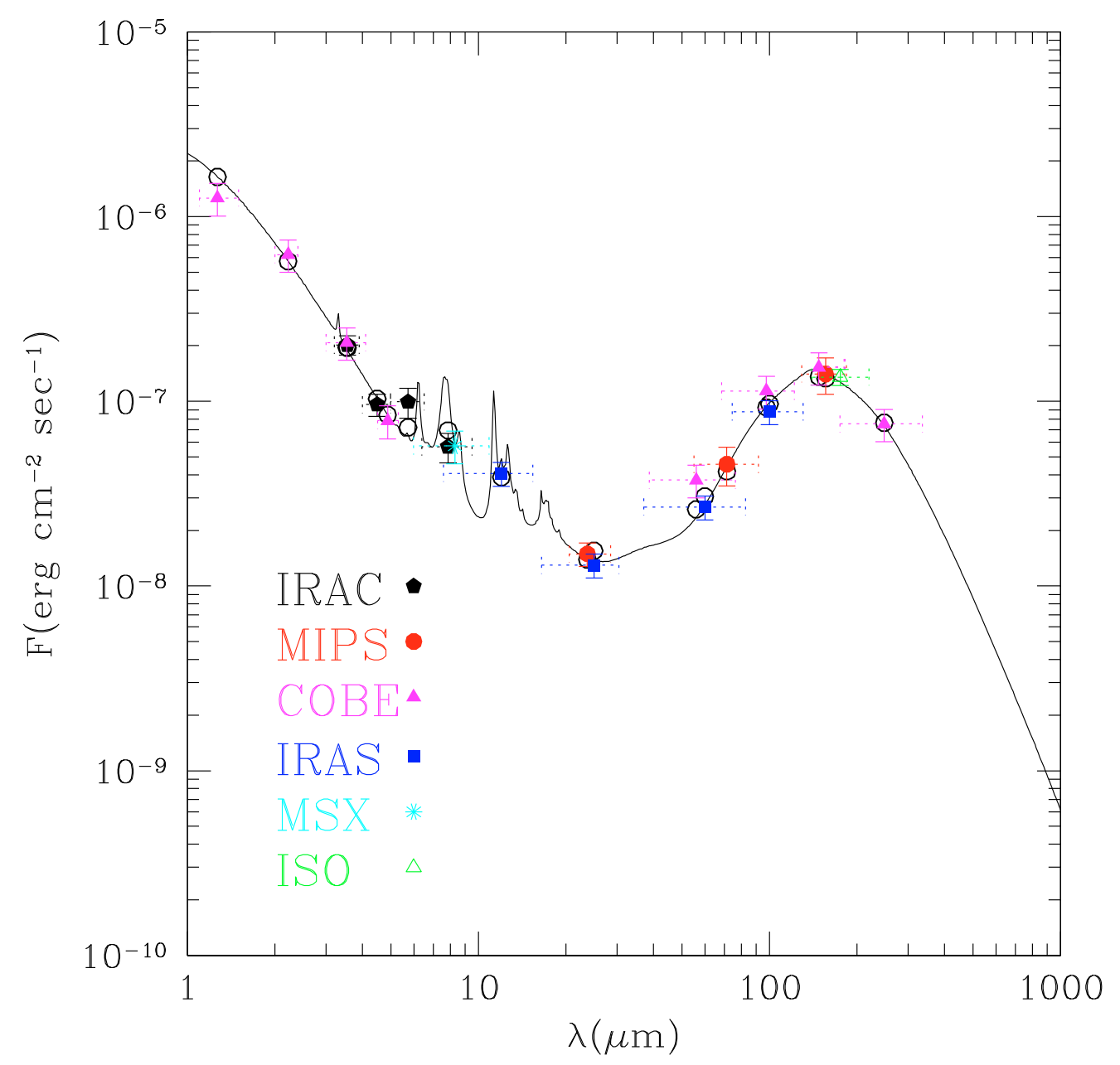

Fig. 3. Infrared spectral energy distribution of M31. Color symbols are measurements obtained with the different instruments indicated in the legend. The continuous line denotes the best-fit model of Draine et al. (2007). Open circles represent the model predicted fluxes after convolution with the instrumental response function, and these are the points that are compared with observations.

increasing the uncertainty in the mass values. Nevertheless, we note that all $\chi^{2}$ curves are highly asymmetric and imply that high mass values are much less tightly constrained than low mass values as explained below.

In Fig. 5, we present the difference between the observations and the values predicted by two different models: (i) the bestfit model (which has $U_{\min }=0.4$, black filled points); (ii) the best-fit model obtained by imposing $U_{\min }=2$ (red open circles). All the measurements reported in Table 2 are considered in the fit, thus the $\chi^{2}$ of these two models are shown in Fig. 4 for the corresponding values of $U_{\min }$ as given by the points connected by the continuous black line. In Fig. 5, only measurements with $\lambda>50 \mu \mathrm{m}$ are considered because this is the spectral region where the major differences are clearly evident. A model should be considered to be consistent with the observations if all the observation minus model differences $(\Delta)$ are consistent with zero, within the observational errors. Focusing attention on the far infrared measurements (FIR) of Spitzer $160 \mu \mathrm{m}$ and COBE $148 \mu \mathrm{m}, 248 \mu \mathrm{m}$ in Fig. 5, we obtained for the bestfit model $\Delta_{148 \mu \mathrm{m}}=0.55 \sigma_{148 \mu \mathrm{m}}, \Delta_{160 \mu \mathrm{m}}=0.24 \sigma_{160 \mu \mathrm{m}}$, and $\Delta_{248 \mu \mathrm{m}}=-0.06 \sigma_{248 \mu \mathrm{m}}$, whereas for the model with $U_{\min }=2$, we obtained $\Delta_{148 \mu \mathrm{m}}=1.74 \sigma_{148 \mu \mathrm{m}}, \Delta_{160 \mu \mathrm{m}}=1.57 \sigma_{160 \mu \mathrm{m}}$, and $\Delta_{248 \mu \mathrm{m}}=2.45 \sigma_{248 \mu \mathrm{m}}$. Thus in this spectral range, the bestfit model with $U_{\min }$ appears to be consistent with the observations at $<0.6 \sigma$, whereas to reconcile observations and model predictions when $U_{\min }=2$ we measure differences $<2.5 \sigma$, and in any case $>1.5 \sigma$. For shorter wavelengths, the two models appear almost equivalent in reproducing the observations, although a slightly worse result is obtained for $U_{\min }=2$. An important feature of Fig. 5 is that for $\lambda \gtrsim 140 \mu \mathrm{m}$, the flux model values for $U_{\min }=2$ are lower than the values of the best-fit model, whereas for $\lambda \lesssim 140 \mu$ m they are systematically higher. This is as expected as the peak emission of the model with $U_{\min }=2$ is shifted towards shorter wavelengths with respect to the best-fit model, as a consequence of the stronger radiation field is heating the dust. Because of FIR model predictions for the case of $U_{\text {min }}=2$, we are already at the limit of predicting an acceptable wavelength distribution, and stronger radiation fields can be discarded. We conclude that on the basis of the diffuse dust emission models of Draine \& Li (2007) and the analysis of the FIR spectrum of M31 the mean radiation field ${ }^{3}$ that is heating the dust in M31 has typically $U<2$. Since the radiation field strnght is inversely proportional to the dust mass estimate, we can also conclude that the mass of the dust in M31 is $\gtrsim 1.1 \times 10^{7} M_{\odot}$, which is the value obtained for the best-fit model with $U_{\min }=2$, assuming a dust-to-gas ratio equal to $1 \%$.

\footnotetext{
${ }_{3}$ In principle, the value of $U_{\text {min }}$ is not consistent with the definition of the mean radiation field given by Draine \& Li (2007), although these two values are essentially equivalent once $\gamma \sim 0$ as seems the case for
} M31. 


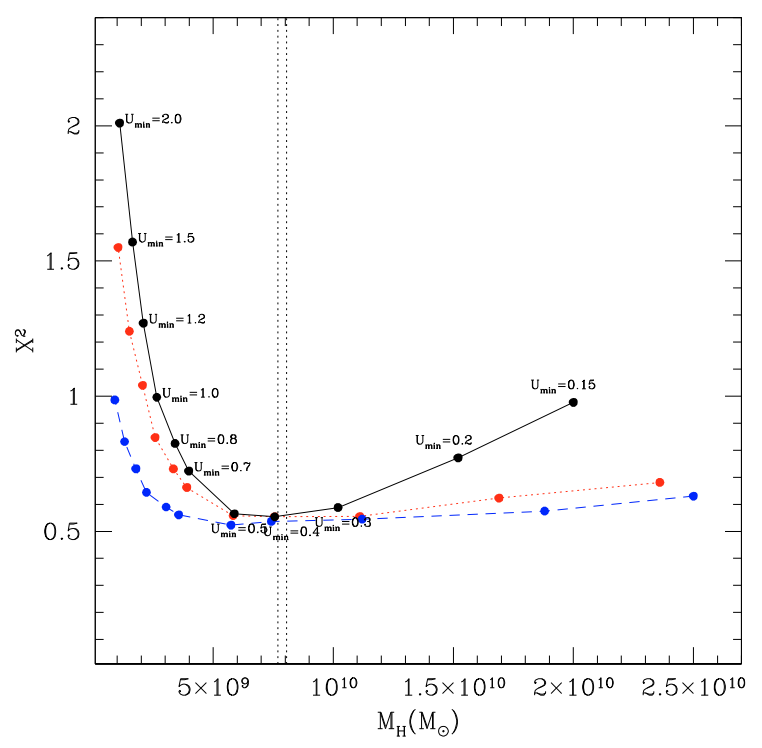

Fig. 4. Minimum $\chi^{2}$ against $M_{\mathrm{H}}$ derived from the model fits, for each fixed value of $U_{\min }$ indicated by the labels. The black points (continuous line) represent the fitting results when considering all the measurements in Table 2 (with the exclusion of ISO and MSX measurements), the red points (dotted line) when neglecting the COBE $248 \mu$ m measurement, and the blue points (dashed line) when neglecting the MIPS $160 \mu \mathrm{m}$ and the COBE $148 \mu \mathrm{m}, 248 \mu \mathrm{m}$ measurements. The vertical dotted lines indicate the range of expected total hydrogen mass values estimated from $\mathrm{HI}$ and $\mathrm{CO}$ maps. Note that the points in this figure are connected by lines for clarity, but the $\chi^{2}$ curve is actually more complex, showing local minima for each value of $U_{\min }$.

The most accurate estimate of the total mass of atomic hydrogen (HI) in M31 is that of Braun et al. (2009), and equals $M_{\mathrm{HI}}=7.33 \times 10^{9} M_{\odot}$. Nieten et al. (2006) provided a mass of molecular hydrogen $M_{\mathrm{H}_{2}}$ equal to $M_{\mathrm{H}_{2}}=3.6 \times 10^{8} M_{\odot}$ (within a radius of $18 \mathrm{kpc}$ ). These estimates infer to a total neutral hydrogen mass of $M_{\mathrm{HI}}+M_{\mathrm{H}_{2}}=(7.69 \div 8.05) \times 10^{9} M_{\odot}$ once considering an uncertainty range for the $\mathrm{CO}$ conversion factor between $X_{\mathrm{HI}}=(2 \div 4) \times 10^{20} \mathrm{~mol} \mathrm{~cm}^{-2}\left(\mathrm{Kkm} \mathrm{s}^{-1}\right)^{-1}$. The mass estimate that we obtained from the best-fit model (considering all the observations in Table $2, M_{\mathrm{H}}=7.6 \times 10^{9} M_{\odot}$ ) is thus respectively $1 \%$ and $5 \%$ lower than the neutral hydrogen mass estimates at the extremes of the uncertainty range mentioned above. Thus, despite the large uncertainty, that we dicussed previously, the best-fit model mass estimate appears to be consistent with $\mathrm{HI}$ and $\mathrm{CO}$ measurements, as shown in Fig. 4. Earlier estimates of the dust mass $\left(M_{\text {dust }}\right)$ in M31 inferred the values equal to $M_{\text {dust }}=3.8 \times 10^{7} M_{\odot}$ (Haas et al. 1998) and $M_{\text {dust }}=1.3 \times 10^{7} M_{\odot}$ (Schmidtobreick et al. 2000), which are fully consistent with our lower limit estimate but, for what has been said so far, they cannot be considered to be more accurate than our best-fit model estimate.

Analyzing a sample of 17 galaxies from the SINGS-SCUBA sample, Draine et al. (2007) showed that in all of these galaxies the value of the mean radiation field derived from the best-fit model was $>2$, with a median value of 4.3 . On that basis, they proposed a restricted fitting procedure for the case in which submillimeter observations are not available, which would require setting $U_{\min }>0.7$ during the fit to avoid overestimating of the dust mass. Nevertheless, as stated by the same authors, this procedure underestimates the dust mass if the radiation field heating the dust is weak. The sample of SINGS-SCUBA galaxies could be biased towards increased associated star formation as

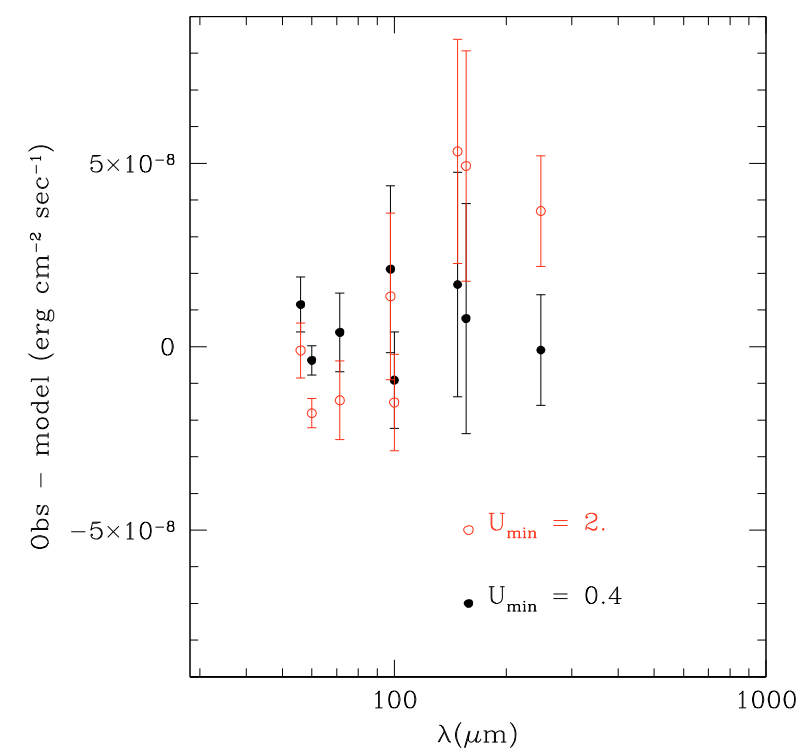

Fig. 5. Observed minus model difference values in flux units for measurements with $\lambda>50 \mu \mathrm{m}$. All measurements reported in Table 2 were used in the fit (with the exclusion of ISO and MSX measurements). Black filled points are the differences obtained considering the bestfit model $\left(U_{\min }=0.4\right)$, whereas red open circles considering the best model among those ones with $U_{\min }=2$ fixed. Errorbars are the uncertainties associated with each measurement as given in Table 2.

recognized by the same authors. As we demonstrated above, for the case of M31, models with $U_{\min }>2$ disagree with the FIR measurements. Moreover, models with a strong UV radiation field imply gas masses far below the range given by neutral hydrogen mass measurements, whereas our best-fit model $\left(U_{\min }=0.4\right)$ is close to reproducing the expected value. This additional constraint excludes models with high $U_{\min }$ and proves that M31 is a galaxy in which the mean UV radiation field heating the dust is very weak. In Sect. 4, we provide an independent demonstration that UV photons are in general not dominating the radiation field in M31, and discuss its implications.

In Fig. 6 (upper panels), we show the correlation between the logarithm of the total IR (TIR) emission and the logarithm of the $24 \mu \mathrm{m}(160 \mu \mathrm{m})$ emission. The TIR was estimated from the $S$ pitzer maps of M31 using the formula given by Draine \& $\mathrm{Li}(2007)$

$$
\begin{aligned}
I_{\mathrm{TIR}}= & 0.95 v I_{v}(8 \mu \mathrm{m})+1.15 v I_{v}(24 \mu \mathrm{m}) \\
+ & v I_{v}(70 \mu \mathrm{m})+v I_{v}(160 \mu \mathrm{m}) .
\end{aligned}
$$

The $\lg (160 \mu \mathrm{m})-\lg (T I R)$ correlation is less scattered (rms = $0.03)$ with respect to the $\lg (24 \mu \mathrm{m})-\lg (\mathrm{TIR})$ correlation $(\mathrm{rms}=$ 0.06). We fitted these data with a simple linear model obtaining

$$
\begin{aligned}
& \lg (\mathrm{TIR})=0.89(0.02) \lg \left(v F_{v}^{n s}[24 \mu \mathrm{m}]\right)-0.1(0.2), \\
& \lg (\mathrm{TIR})=1.1(0.01) \lg \left(v F_{v}^{n s}[160 \mu \mathrm{m}]\right)-1.3(0.1)
\end{aligned}
$$

Although, as shown below, the correlation between the $24 \mu \mathrm{m}$ $(160 \mu \mathrm{m})$ and the TIR emission is not strictly linear, these results (in particular the calibration relation between the $\lg (24 \mu \mathrm{m})$ and the $\lg$ (TIR) variables) becomes useful later in Sect. 5, and is sufficiently accurate for our purposes.

In Fig. 6 (bottom panels), we show the $24 \mu \mathrm{m} / \mathrm{TIR}$ (160 $\mu \mathrm{m} / \mathrm{TIR})$ ratios against the TIR intensity. As the TIR increases, the $24 \mu \mathrm{m} / \mathrm{TIR}$ ratio increases, but is restricted to typically between $5 \%-7 \%$. In contrast, the $160 \mu \mathrm{m} / \mathrm{TIR}$ decreases 

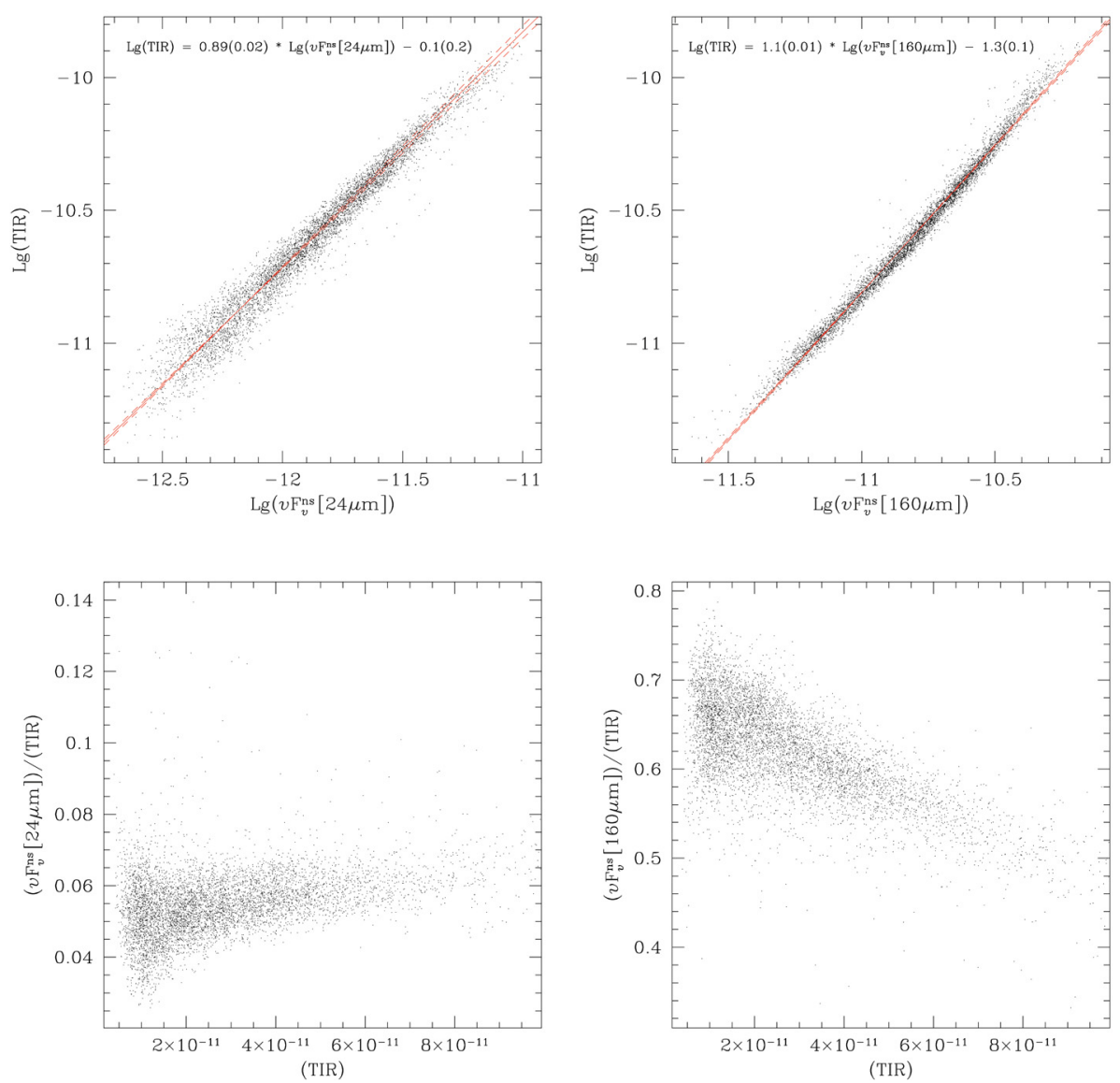

Fig. 6. Upper panels: Correlations between the logarithm of the TIR emission and the logarithm of the $24 \mu \mathrm{m}(160 \mu \mathrm{m})$ emission. Lower panels: correlations between the $24 \mu \mathrm{m} / \mathrm{TIR}$ $(160 \mu \mathrm{m} / \mathrm{TIR})$ emission ratio and the TIR emission. Only regions with $S / N>1$ are considered.

from $\sim 65 \%$ to $\sim 50 \%$. This demonstrates that overall the infrared spectrum of M31 is dominated by cold dust emission in the FIR, although the contribution of hotter dust components tends to increase for higher TIR emission.

\subsection{PAHs abundance, $\gamma$}

Figure 7 , shows the ratio $R_{8}$ of the $8 \mu \mathrm{m}$ flux (normalized to the total infrared emission) against the ratio $R_{71}$ of $71 \mu \mathrm{m}$ flux to $160 \mu \mathrm{m}$ flux. As proposed by Draine et al. (2007), this diagram can be used to estimate the abundance of PAHs. We used it to illustrate the uncertainty in our fit by comparing the result obtained from the total integrated emission of M31 to that obtained in the single regions of our maps, resampled to the $160 \mu \mathrm{m}$ resolution and using exclusively the regions with $S / N>3$. Uncertainties were calculated as explained in Sect. 2. This selection restricted us to the study of the ring-spiral structure. The PAHs are stochastically heated by single photons, thus to derive their abundance one compares the observations with theoretical models with $\gamma=0$ (Draine et al. 2007, where $\gamma$ is the parameter that regulates the power-law intensity radiation field in Eq. (3)). In Fig. 7, we show the theoretical predictions as functions of the different amount of PAHs and the minimum radiation field intensity $U_{\min }$. These results imply that models with large abundances $(>3.19 \%)$ and rather low $U_{\min }$ are favored when comparing with the data. The measurement associated with the integrated infrared emission (blue point) is not at the center of the distribution of points produced by the analysis of the pixel-topixel analysis, and in particular is biased towards lower values of $R_{8}$. Since our analysis is restricted to the spiral-ring structure, this result suggests that in the inter-arm regions and/or toward the bulge, the abundance of PAHs could be lower than we

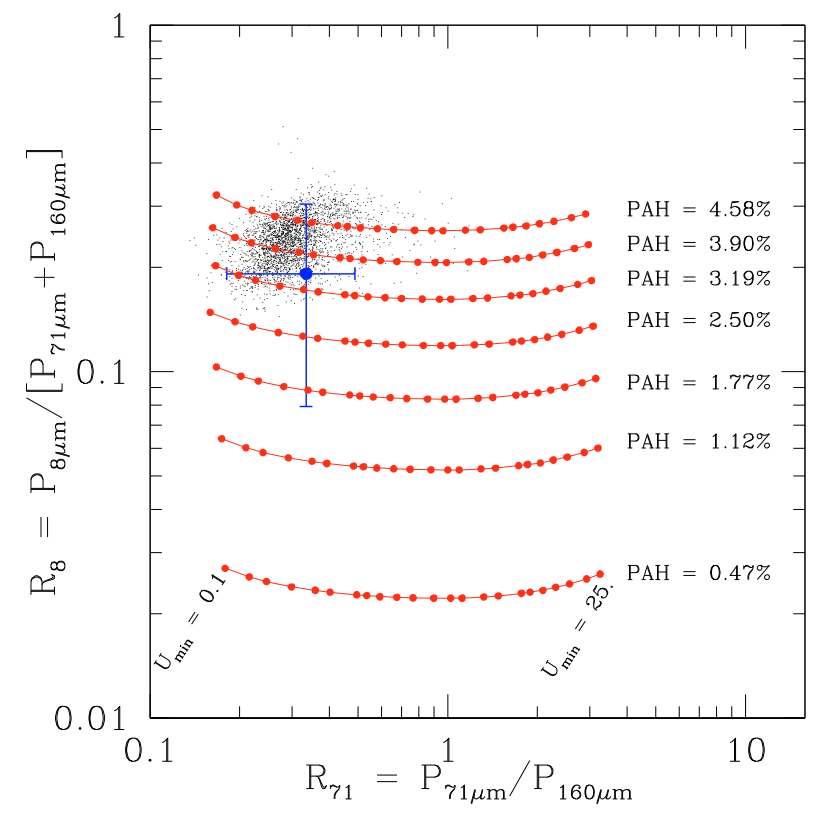

Fig. 7. Ratio $R_{8}$ of the $8 \mu \mathrm{m}$ flux normalized to FIR emission against the ratio of $71 \mu \mathrm{m}$ to $160 \mu \mathrm{m}$ flux $R_{71}$. The blue point is the ratio corresponding to the total integrated flux measurement of M31. Each horizontal red line shows the values predicted by the models of Draine \& $\mathrm{Li}$ (2007) for different PAH abundances and values of the minimum radiation field intensity $U_{\min }$.

derived, but an accurate analysis of the distribution of these particles across the entire disk of M31 is beyond the purpose of this work. 
In Fig. 8, we show the ratio $R_{24}$ of the $24 \mu \mathrm{m}$ flux (normalized to the total infrared emission) against the ratio $R_{71}$, a diagram similar to Fig. 7, but for the $24 \mu \mathrm{m}$ map, which is more reliable diagnostic to the radiation field intensity (Draine et al. 2007). In this case, we considered only models with PAHs abundances $>3.19 \%$, since for the analyzed regions these are the models that agree more closely with observations (Fig. 7). For the bulk of the points, and the total integrated flux measurement, $\gamma$ can in general be considered $\leq 0.05$. Using the equation

$f_{\mathrm{PDR}}=1.05\left(R_{24}-0.14 R_{8}-0.035\right)^{0.75}$

taken from Draine $\&$ Li (2007) and the observed ratios $R_{8}$ and $R_{24}$ defined above, we determined that the fraction of the total dust luminosity radiated by dust grains in regions with $U>10^{2}$ $\left(f_{\mathrm{PDR}}\right)$ is lower than $\sim 4 \%$.

\section{Which sources are responsible for the dust heating in M31?}

The analysis of the infrared spectrum presented in Sect. 3 allows to conclude that the infrared spectrum of M31 can be explained by diffuse dust emission. Nevertheless, on the basis of that, we cannot understand which stellar population is responsible for the dust heating process. In particular, we want to know where the dust in M31 is predominantly heated by young populations (age $<1 \mathrm{Gyr}$ ) or where older populations (age $>1$ Gyr) play also an important role in the dust-heating process. As shown below, the answer to this question is of crucial importance to the measurement of several important parameters, e.g., dust attenuation and the SFR.

In the following, we used the observational maps of M31 resampled to $40^{\prime \prime} / \mathrm{px}$ as described in Sect. 2, and considered all the regions in which the $S / N>1$ in all the maps.

It should also be noted that the results we obtained are based on the theoretical models of Kong et al. (2004) and Cortese et al. (2008). It is worth saying that alternative interpretations may exist of the observational data we present. In particular, models considering modifications of the canonical attenuation laws have been proposed (e.g., Inoue 2005, 2006, see later in this section).

The model of Kong et al. (2004) is based on the Bruzual \& Charlot (2003) population synthesis code, and assumes an exponential star formation history (SFH) and a power-law absorption law, distinguishing between young $\left(<10^{7} \mathrm{yr}\right)$ stars embedded in their birth clouds and older stars that have migrated into the ISM. The model of Cortese et al. (2008) is also based on the Bruzual \& Charlot (2003) population syntesis code, but assumes an LMC attenuation law, and that both stars and dust were homogeneously distributed in a plane-parallel (sandwich) geometry. They also adopted a star formation history "a la Sandage" in the formalism of Gavazzi et al. (2002)

$S F R(t, \tau)=\frac{t}{\tau^{2}} \exp \left(-\frac{t^{2}}{2 \tau^{2}}\right)$,

where SFR is the star formation rate per unit mass, $\mathrm{t}$ is the galaxy age (in Gyr, assuming $t=13 \mathrm{Gyr}$ at present epoch), and $\tau$ is the time (in Gyr) at which the star formation rate reached its highest value during the entire galaxy history (note that in this notation, the present age of the stellar populations born at time $t=\tau$ is given by age $=13 \mathrm{Gyr}-\tau$ ). As stated by the same authors, $\tau$ should be considered to be a proxy for the shape of the SED rather than being used to derive an exact indication of the age the underlaying stellar populations. For each value of $\tau$, we calculated the time $t^{*}$ at which the SFR reaches half of its maximum
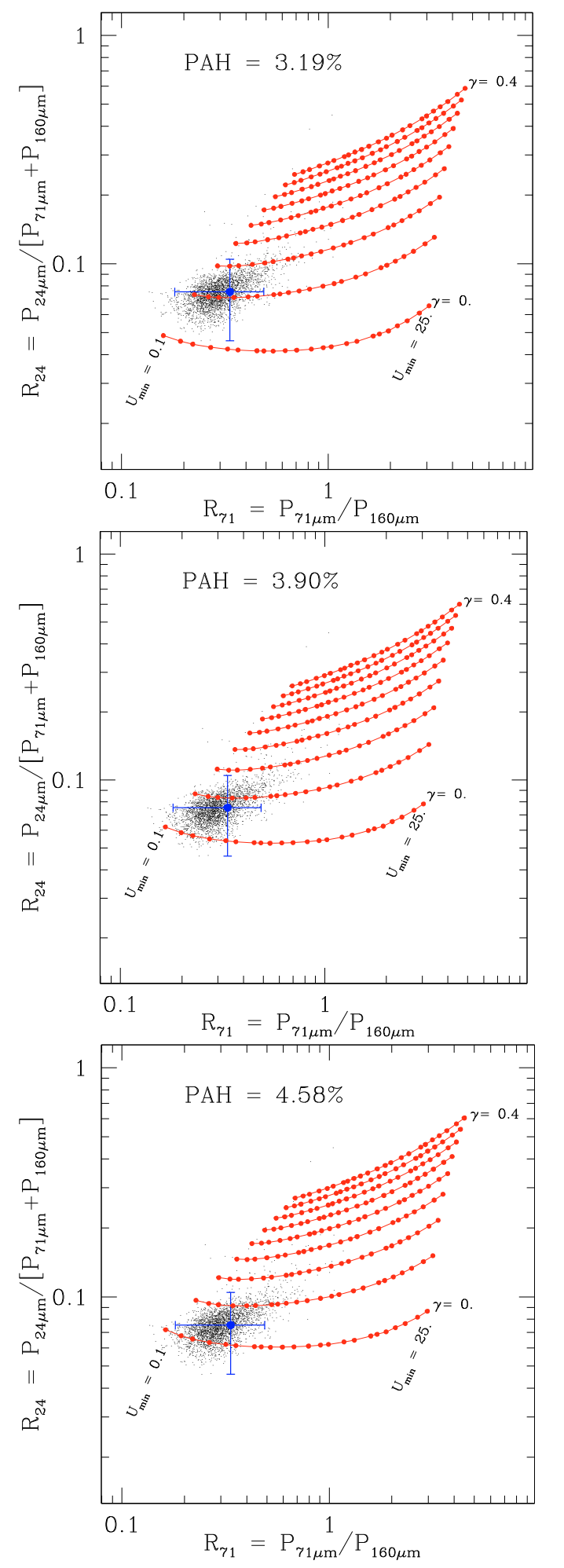

Fig. 8. Ratio $R_{24}$ of the $24 \mu \mathrm{m}$ flux normalized to FIR emission against $71 \mu \mathrm{m}$ to $160 \mu \mathrm{m}$ flux ratio $R_{71}$. The blue point is the ratio corresponding to the total integrated flux measurement of M31. Each horizontal red line shows the ratio values predicted by the models of Draine \& $\mathrm{Li}$ (2007) for different values of the minimum radiation field intensity $U_{\min }$ and of the $\gamma$ parameter (see text). The three panels show the results for models with different abundances of PAHs as indicated by the labels.

value (for $t>\tau$ ) over the whole galaxy history. Also the age of the stellar populations corresponding to $t=t^{*}$ must not be considered in a strict manner but can be considered as an indication 


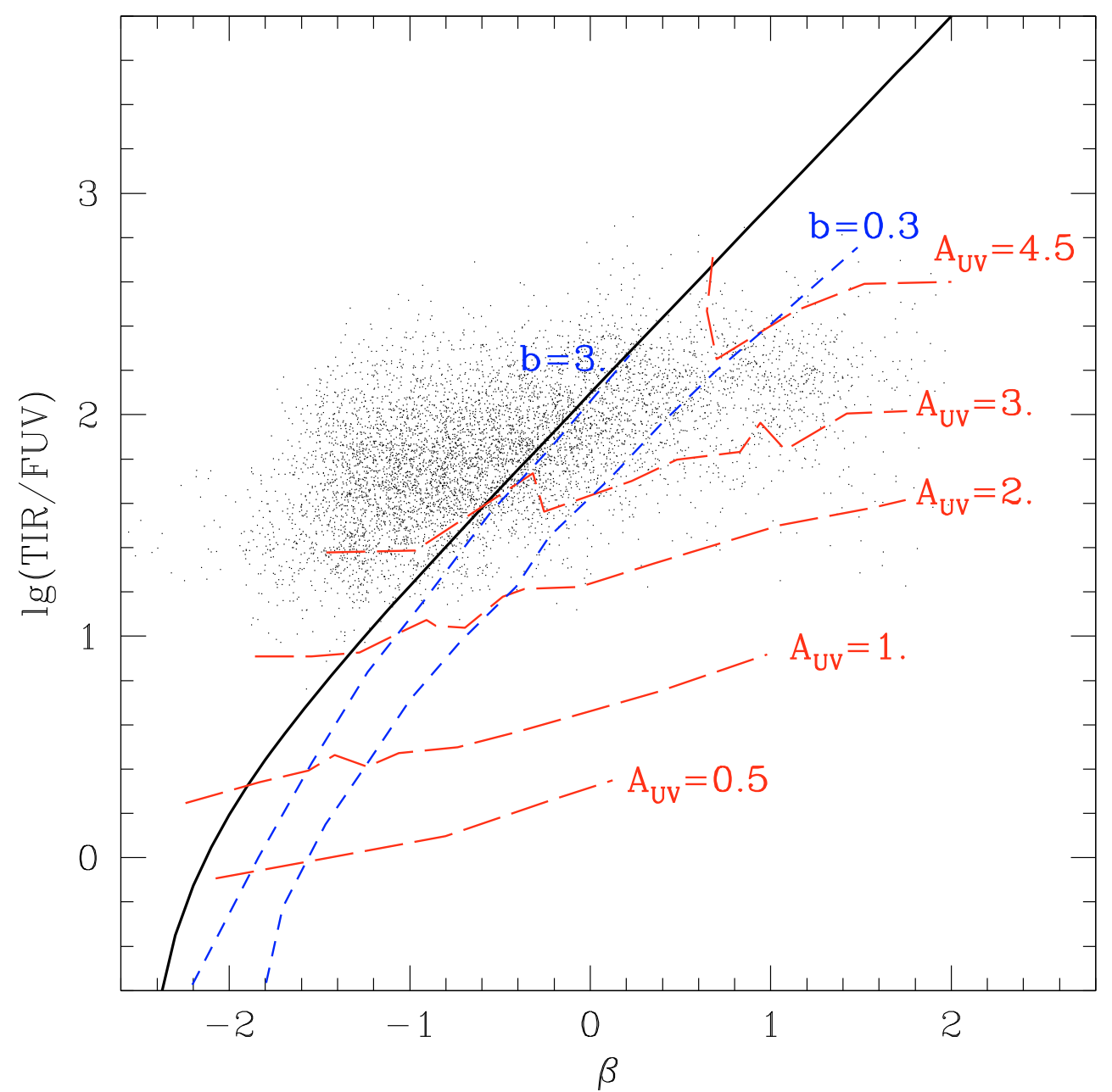

Fig. 9. (TIR/FUV) logarithmic ratio against $\beta$, the UV-slope, for each region with $S / N>1$ analyzed in the $40^{\prime \prime} /$ px resolution maps. The thick continuous line denotes the best fit of Kong et al. (2004) for their sample of starburst galaxies. The long dashed red lines and the short dashed blue lines are constant attenuation and constant ratio of present to past-averaged star formation rate lines as given by Kong et al. (2004).

of the age of the youngest stellar populations contributing significantly to the radiation of the SED, given that the contribution of future generations of stars becomes increasingly less important in explaining the observed SED. For example, for $\tau=8 \mathrm{Gyr}$ we obtain $t^{*} \approx 13.5 \mathrm{Gyr}$. Assuming for the present epoch $t=13 \mathrm{Gyr}$ as in Cortese et al. (2008), we can expect that young populations (age $<1$ Gyr) contributed significantly to the observed SED. For $\tau=5$ Gyr, we obtain instead $t^{\star} \approx 9.6 \mathrm{Gyr}$, which implies that stellar populations contributing to the SED should have an age $>3$ Gyr.

We also note that the model predictions should not be considered reliable in regions where old stars are likely to dominate the UV flux (mostly in the bulge region for M31).

We first investigated the TIR/FUV versus $\beta$ (the UV slope) relationship for the analyzed regions as shown in Fig. 9. The solid line represents the fit obtained by Kong et al. (2004) for the 50 starburst galaxies in their sample, which represents the socalled IRX $-\beta$ relationship for starburst galaxies (Meurer et al. 1999). We adopted the same definition as Kong et al. (2004) in calculating $\beta$

$\beta=\frac{\lg \left(\bar{f}_{F U V}\right)-\lg \left(\bar{f}_{N U V}\right)}{\lg \left(\lambda_{F U V}\right)-\lg \left(\lambda_{N U V}\right)}$,

where $\lambda_{F U V}=1516 \AA$ and $\lambda_{N U V}=2267 \AA$ are the effective wavelengths of the far-ultraviolet and near-ultraviolet filters onboard GALEX and $\bar{f}_{F U V}, \bar{f}_{N U V}$ are the mean flux densities (per unit wavelength) through these filters.

The small black points are the values of the TIR/FUV and $\beta$ obtained in the analyzed regions of M31. A positive correlation is visible, but does not follow the relation found for starburst galaxies. As can be naively expected, going towards negative slopes and bluer colors our observations imply similar TIR/FUV and $\beta$ values with respect to the starburst galaxies relationship, whereas redder regions having differently showing typically lower values of TIR/FUV for equivalent $\beta$. This agrees with Kong et al. (2004), who showed the results coming from the analysis of their sample of normal star-forming galaxies as well and found that these objects typically have lower values of TIR/FUV for fixed $U V$-color than predicted by the $(I R X-\beta)$ relation, with a large scatter of values. Other authors have questioned the application of the $(I R X-\beta)$ relationship to normal star-forming galaxies, suggesting that these systems have lower dust attenuations than can be inferred from the $(I R X-\beta)$ relation (Salim et al. 2007).

Following the notation of Kong et al. (2004), we plot in Fig. 9 the lines of constant ratio of present to past-averaged star formation rate assuming an exponentially declining star formation history ( $b$ parameter, blue short-dashed lines), and constant attenuation ( $\mathrm{A}_{\mathrm{UV}}$, red long-dashed lines). This comparison suggests that in the M31 regions, population gradients are present but there are 
similar amounts of dust attenuation, our observational points being elongated along the direction indicated by the bands of equal attenuation.

Since color variations seem to be caused by a spread of ages, it is more convenient to adopt a wider color baseline such as the $\left(\mathrm{FUV}-i_{\mathrm{SDSS}}\right)^{4}$ color. Cortese et al. (2008) provided a detailed discussion of the age dependence of the TIR/FUV ratio, colors, and attenuations. In accordance with their results, for (FUV $\left.i_{\text {SDSS }}\right) \sim 4.3$, the energy absorbed by the dust at $\lambda<4000 \AA$ is approximately equal to the energy absorbed at $\lambda>4000 \AA$ (as inferred from their Fig. 2 and Table 1). This corresponds to $t^{\star} \simeq 8.5 \mathrm{Gyr}$ as defined above, and thus the stellar populations contributing to the SED have an age $>4.5$ Gyr.

In Fig. 10, we show the TIR/FUV ratio against the (FUV $i_{\text {SDSS }}$ ) color, for regions with $S / N>1$ across the $10 \mathrm{kpc}$ ring $(8 \mathrm{kpc}<r<12 \mathrm{kpc})$. The positive correlation between these variables is also clearly visible in this diagram. Moreover, for the color range $3.5<$ (FUV $\left.-i_{\text {SDSS }}\right)<7$ it is clearly evident that in a large number of regions, old populations dominate the dust heating process. As shown in Fig. 10 (lower left panel), the mean $A_{\mathrm{FUV}}$ in the analyzed regions of M31 derived with this method is $A_{\mathrm{FUV}}=2.1 \pm 0.4 \mathrm{mag}$, where the error is the standard deviation. If the starburst scenario is considered, we obtain the result shown in the lower right panel of Fig. 10, where the mean attenuation is $A_{\mathrm{FUV}}=4.2 \pm 1.0 \mathrm{mag}$. Neglecting the dust heating age dependence would imply a mean attenuation that is $100 \%$ higher and a scatter $150 \%$ larger, since in this case the large color changes would be attributed entirely to the reddening.

In Fig. 10, we overplotted two lines of constant attenuation corresponding to $A_{\mathrm{FUV}}=2$ and $A_{\mathrm{FUV}}=3$ as indicated by the labels. The shape of these lines reflects the age dependence of the dust heating as modeled by Cortese et al. (2008). For blue $\left.\left(\mathrm{FUV}-i_{\mathrm{SDSS}}\right)<4\right)$ or red colors $\left(\mathrm{FUV}-i_{\mathrm{SDSS}}\right)>7$, the attenuation lines tend to be parallel to the color axis, and thus independent of the color. This because in these color ranges, the intrinsic spectral energy distribution of the populations heating the dust is not expected to change appreciably with age towards the very young (blue) or very old (red) side. On the other hand, in the range $4<\left(\mathrm{FUV}-i_{\mathrm{SDSS}}\right)<7$, which corresponds to most of our observations, age variations contribute significantly to color changes, and older populations appear intrinsically redder thus shifting the TIR/FUV ratio towards higher values for equal amounts of attenuation, than to bluer regions. In the following, we tried to disentangle the effects of age and reddening on the observed colors and TIR/FUV ratios.

At first, we converted the colors to the values of $\tau$ (the time, expressed in Gyr, at which the star formation rate reached its highest value over the whole galaxy history, assuming $13 \mathrm{Gyr}$ at present epoch) by means of

$\lg (\tau)=-0.073\left(\mathrm{FUV}-i_{\mathrm{SDSS}}\right)+0.96$,

taken from Table 2 of Cortese et al. (2008). The colors in Fig. 10 are defined as a function of the value of $\tau$. We found that $83 \%$ of the analyzed regions across the spiral-ring have (FUV $\left.-i_{\text {SDSS }}\right)>$ 4.3 and thus values of $\tau<4.4$ Gyr. Hence, according to the results of Cortese et al. (2008) in $83 \%$ of the analyzed regions, the dust absorbs more than $50 \%$ of the energy at $\lambda>4000 \AA$. As stated above, this implies that in these regions stellar populations responsible for the dust heating should be at least a few Gyr

\footnotetext{
${ }^{4}$ We decided to use here the (FUV $-i_{\text {SDSS }}$ ) color because the 2MASS images of M31 obtained during the 2MASS Extended Survey did not appear sufficiently deep for our purposes.
}

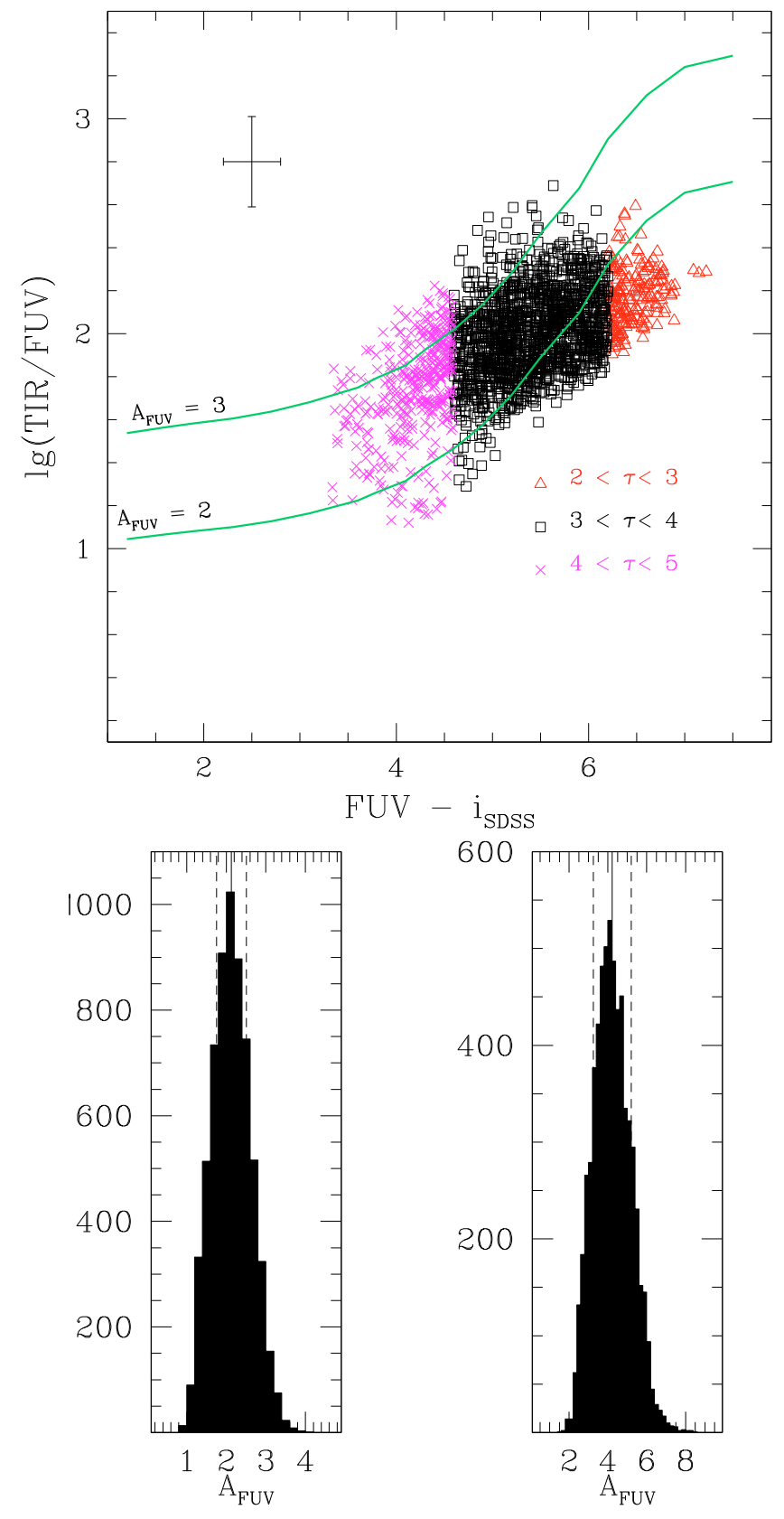

Fig. 10. Upper panel: (TIR/FUV) logarithmic ratio against (FUV $\left.i_{\text {SDSS }}\right)$ color for regions with $S / N>1$ across the $10 \mathrm{kpc}$ ring $(8 \mathrm{kpc}<$ $r<12 \mathrm{kpc}$ ). The colors denote the different values of $\tau$ (the time expressed in Gyr at which the star formation rate reaches the highest value over the whole galaxy history, assuming $13 \mathrm{Gyr}$ at present epoch) obtained for each region following the recipes of Cortese et al. (2008). The two solid lines are two lines of constant attenuation. The errorbar on the top-left corner shows the mean observational errors. Lower panels: histograms of ultraviolet attenuations derived following the recipes of Cortese et al. (2008, left panel) and assuming the starburst scenario (right panel). Mean values and $1 \sigma$ uncertainties are denoted by solid and dashed vertical lines and are $A_{\mathrm{FUV}}=2.1 \pm 0.4 \mathrm{mag}$ and $A_{\mathrm{FUV}}=4.2 \pm 1.0 \mathrm{mag}$ for left and right panel respectively.

old. This could provide good agreement with the low mean intensity of the radiation field that we found from the analysis of the infrared spectrum of M31, although a small value of $U$ could also be obtained by young stars with a low SFR. It is important to recall that these results do not imply that younger populations are not present at all, but rather that their contribution is 


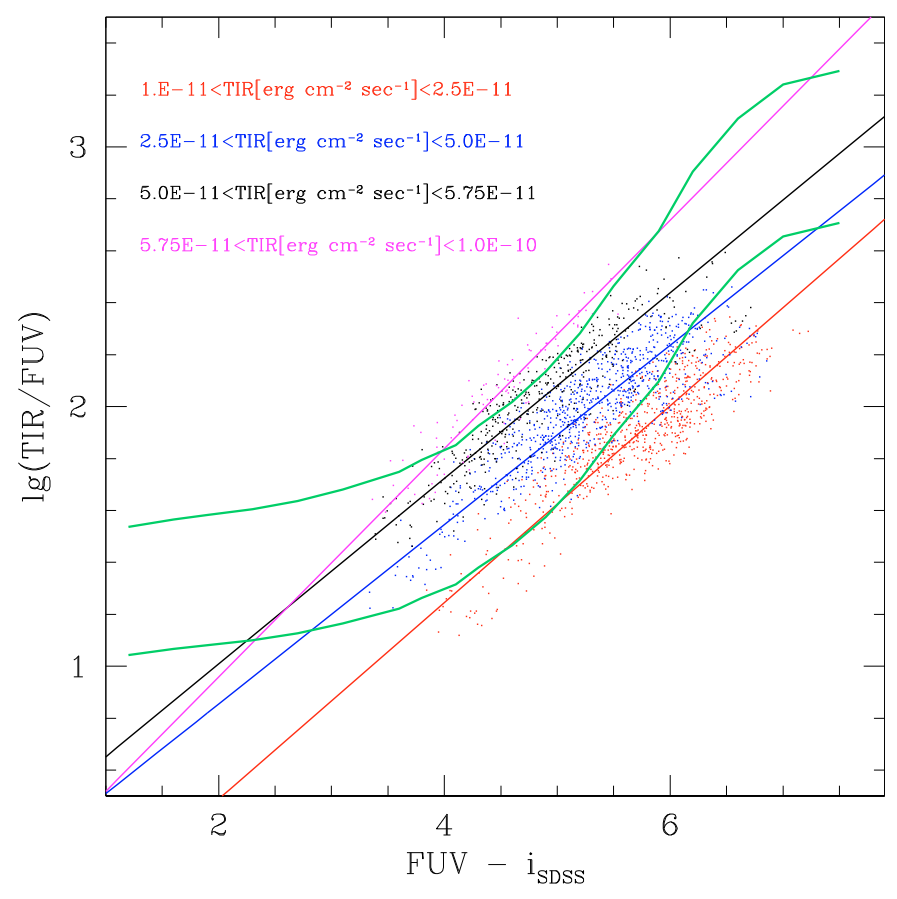

Fig. 11. TIR/FUV logarithmic ratios versus (FUV $-i_{\mathrm{SDSS}}$ ) colors for regions with $S / N>1$ across the $10 \mathrm{kpc}$ ring $(8 \mathrm{kpc}<r<12 \mathrm{kpc})$. Different colors and labels denote regions of constant TIR emission. Red, blue, black, and magenta lines indicate the best-fit linear regression models of the data in each TIR emission interval. Green curves are two constant attenuation lines accordingly to the recipes of Cortese et al. (2008) as shown in Fig. 10.

not dominant in these regions. If, in contrast, one tries to explain the observed colors by assuming that only young populations (age $<1$ Gyr) heating the dust, one should also fit much larger attenuations for normal galaxies, as demostrated above and for the same distribution of stars and dust as in Cortese et al. (2008). Otherwise it is clear that in order to confirm these results, more accurate models are needed. Some other considerations regarding the limitation of the Cortese et al. (2008) approach are presented briefly below and in the next Section.

From another point of view, we show in Fig. 11 the same diagram of Fig. 10, separating regions of low and high TIR emission as indicated by the labels and the colors. We considered 4 equally sized bins of TIR emission in the range $10^{-11}-10^{-10} \mathrm{erg} \mathrm{cm}^{-2} \mathrm{~s}^{-1}$. From Fig. 11, it appears that towards redder colors along the regions of constant TIR emission, the TIR/FUV ratio increases. Since the TIR emission is constant, the FUV emission must decrease. Nevertheless, if the lower FUV emission was caused by higher attenuations, the TIR emission should have increased accordingly, which is not observed. Thus, the color change must be caused by an intrinsic lower emission in the FUV band, which could be indicative of a population gradient. Strictly speaking, this interpretation is valid for an homogeneous layer of stars embedded in an homogeneous optically thin layer of dust. In a real galaxy, dust and stars (especially recently formed stars) are likely to have more clumpy and inhomogeneous distributions. Otherwise, studying two of the more conspicuous and more discrete dust clouds listed in the M31 atlas (Hodge 1980); Hodge \& Kennicutt (1982) derived average extinctions of $A_{\mathrm{B}}=0.35 \mathrm{mag}$ (with a maximum value of $A_{\mathrm{B}}=0.60 \mathrm{mag}$ ) and $A_{\mathrm{B}}=0.43 \mathrm{mag}$ (maximum $A_{\mathrm{B}}=0.76 \mathrm{mag}$ ) for $\mathrm{D} 307$ and $\mathrm{D} 441$, respectively, indicating that the majority of all the other clouds should have smaller amounts of extinction. Other studies confirm the idea that the interstellar medium in M31 is generally optically thin. Fan et al. (2007) derived reddening values towards 443 M31's catalogued globular clusters, find that more than half of them are affected by a reddening $\mathrm{E}(\mathrm{B}-\mathrm{V})<\sim 0.2 \mathrm{mag}$ with an average value of $\mathrm{E}(\mathrm{B}-\mathrm{V})=0.28_{-0.14}^{+0.23}$ (see also Barmby et al. 2000). Regarding the homogeneity of the dust distribution, Inoue $(2005,2006)$ investigating the attenuation law for clumpy spatial distributions of dust and (young) stars, proposed different explanations of the redder $U V$ colors of normal galaxies with respect to starburst galaxies of fixed FIR to FUV ratios, which are not based on population gradients. These alternative models can be summarized as: (i) models where the attenuation law has a stronger dependence on wavelength than the canonical attenuation laws as a result of an age-selective attenuation of young stars in clumpy structures with respect to old stars; (ii) models where the attenuation law has no attenuation bump at $2175 \AA$; (iii) models with a bump at $2175 \AA$, but of smaller albedo at shorter wavelengths (except for the bump range). While we did not try to apply these models to our data, we observe that at least the first two scenarios do not seem to be applicable to M31. Bianchi et al. (1996) found that the M31 extinction curve is very similar to the average Galactic extinction law and a possible reduction in the $2175 \AA$ bump is significant only at the $1 \sigma$ level. Similar results were also reached in previous studies (e.g., Walterbos \& Kennicutt 1988). As for the third class of models, further studies of the wavelength dependence of the albedo are required. We also note that these considerations do not imply that clumpy structures with embedded young stars do not exist in M31, but it could be that most of the dust in M31 is not located in these structures. From counts of dark nebulae across the disk of M31, Hodge \& Kennicutt (1982) found that the major dust lanes visible in the optical should account for $\sim 15 \%$ of the total dust mass content. Nieten et al. (2007) measured a strong correlation between the most prominent dust lanes and dense molecular clouds traced by strong $\mathrm{CO}$ emission lines, but concluded that molecular gas in M31 represents only 7\% of the total neutral gas content in M31, and that dust also appears correlated with atomic gas in M31, which is distributed in more extended regions outside of the densest clouds in the spiral-ring structure. In Sect. 3.1, we found that only a fraction $<7 \%$ of the TIR originates in the hot dust component emission at $24 \mu \mathrm{m}$ associated with young starforming regions. Finally, it is unlikely that a large fraction of the M31's dust mass is in very cold dark clouds $(T<16 \mathrm{~K})$, as the total M31 dust mass we derived in Sect. 3.1 from dust diffuse emission models was close $(1 \%-5 \%)$ to the estimate based on neutral gas measurements. We conclude that the interpretation of Fig. 11 in terms of population gradients must be considered carefully and is in need of further investigation, but it appears reasonable for regions dominated by diffuse dust emission away from large star-forming complexes (in this case, we recall the criteria that we applied in Sect. 2 against the selection of HII regions). The attenuation within bands of equal TIR emission appears to decrease towards redder colors accordingly to the predictions of Cortese et al. (2008). This could also be caused by a population gradient, since older populations would tend to be intrinsically less attenuated than younger ones, because of their intrinsically redder spectral energy distribution, at least in the region where the age effect is maximal $4<\left(\mathrm{FUV}-i_{\text {SDSS }}\right)<7$. We note nevertheless that the observed color could be also biased towards redder values especially in the bulge region where the geometry of the dust and star clearly deviates from the simple sandwich model of Cortese et al. (2008). We discuss this potential bias in 


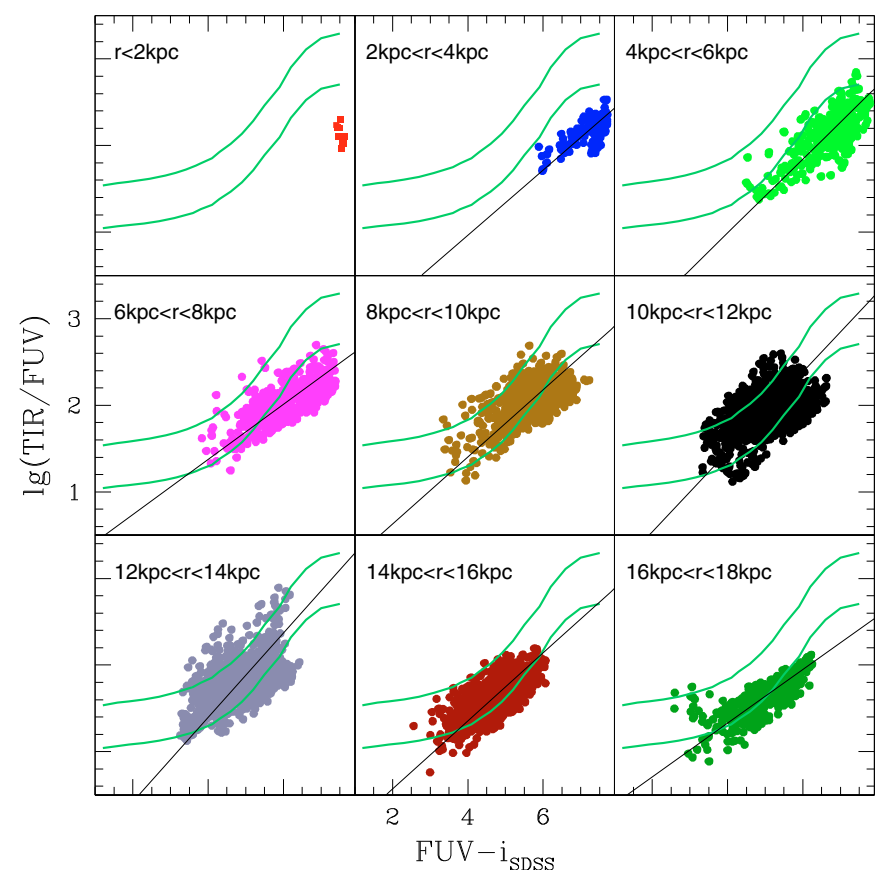

Fig. 12. Logarithmic TIR/FUV ratios against FUV - $i_{\text {SDSS }}$ colors for regions inside each annulus. Thin black solid and dashed lines are bestfit linear models of the data as explained in the text. Green curves are constant attenuation lines accordingly to the recipes of Cortese et al. (2008) as shown in Fig. 10.

more details in the next section. Another interesting point about Fig. 11, is that towards higher TIR/FUV ratios, the TIR emission increases (whereas the FUV emission remains almost constant), which should infer higher attenuation values because of the large amount of absorbed energy for a fixed observed FUV energy, which is what the model predicts. We have thus the following interpretation of the diagram in Fig. 11. While the large color variation of the analyzed regions seems to be caused by a population gradient in the stars heating the dust, the spread in the TIR/FUV values for a fixed color seems instead to be caused by the differential reddening of regions with similar underlaying stellar populations.

We also investigated the spatial distribution of the $A_{\mathrm{FUV}}$ across the disk of M31. We considered nine $2 \mathrm{kpc}$-wide radial bins from the center of M31 out to $18 \mathrm{kpc}$ as shown in Fig. 2, and imposed a $S / N>1$. In Fig. 12, we present the logarithmic TIR/FUV ratios versus the (FUV $-i_{\text {SDSS }}$ ) colors obtained in each radial bin. In general, it appears that these observable quantities are positively correlated across the entire disk of M31. Only in the innermost annulus was it impossible to verify the positive correlation of these variables because of the few regions analyzed. We fit the $\lg (\mathrm{TIR} / \mathrm{FUV})$ ratios versus $\left(\mathrm{FUV}-i_{\mathrm{SDSS}}\right)$ colors in each radial bin with a linear model $\lg (\mathrm{TIR} / \mathrm{FUV})=a\left(\mathrm{FUV}-i_{\mathrm{SDSS}}\right)+b$. We considered separately the uncertainties along the $x$ and $y$ axis directions and considered the mean value of the derived parameters and their semidifference as estimates of the best-fit model parameters ( $a$ and $b$ ) and uncertainties. In Table 3, we report the result of the fit and other useful quantities.

As also shown in Fig. 13, the mean attenuation in each radial bin varies reaching a maximum near the $10 \mathrm{kpc}$ ring, while it seems to decrease faster towards the inner regions of M31 than in the outer regions.
These results are also shown in the color maps of Fig. 14. As dicussed above twhile he model adopted here could be less reliable in the inner part of the galaxy because of deviations in the dust and star distribution from the assumed geometry, the trend in and the values of the mean attenuations derived with this method agree with previous studies as discussed in the next section.

The existence of a correlation between the TIR/FUV ratios and the colors that we have found needs a final remark. It is important to recall that the theoretical calculations of Kong et al. (2004) and Cortese et al. (2008) do not necessarily imply the existence of any correlation between these observables. Nonetheless, we should consider that in this case we are study regions inside one galaxy, and thus we should expect a more uniform and homogeneous behavior.

\section{Discussion}

The models of Cortese et al. (2008) assume a simple planeparallel (sandwich) geometry for the dust and star distribution. Even if the fractional scale height between dust and the stars is allowed to vary with wavelength, no radial dependence is assumed. The method is based solely on the relationship between two observed emission ratios, and is therefore expected to be sensitive to light contamination from distant sources and variations in geometry. Since, if the geometry is not plane parallel the radiation field experienced by the dust can significantly differ from that naively measured by the outside observer, the observed color from which the age of the underlying population is derived could be biased towards redder values in regions where older stellar populations are less embedded in the dust. This bias should be strongest in the inner regions of the galaxy as the result of bulge light contamination. Regions with $r<8 \mathrm{kpc}$ in Fig. 12 have redder colors and higher TIR/FUV ratios than the outer regions. For $r>12 \mathrm{kpc}$, the color range remains almost constant, although the TIR/FUV ratio decreases. In the presence of the above mentioned observational bias, the value of the attenuation derived from the model may have been underestimated in the inner regions. Moreover, in the innermost radial bin $(r<2 \mathrm{kpc})$, old stars can produce a significant fraction of the GALEX-UV emission, and thus the model prediction is likely to be unreliable in that region.

To estimate how the integrated light along the line of sight could have affected our analysis and consequently biased the derived attenuations, we compared the (FUV $-i_{\text {SDSS }}$ ) color along the major and minor axis, as shown in Fig. 15. Each point in these figures is the average value of the colors (attenuations) inside a $200^{\prime \prime}$-wide $(0.75 \mathrm{kpc})$ stripe centered on the major and minor-axis corresponding to each of the $2 \mathrm{kpc}$ annular regions shown in Fig. 2. Any effect of bias should be more evident along the minor than the major axis because of the inclination of M31. At the same galactocentric radius of the disk, the line of sight passes through more central regions of the bulge along the minor axis than along the major axis. If the described bias affects the derived parameters, it is expected that at the same galactocentric radius the adopted method will predict lower attenuations along the minor axis than on the major axis. We calculated the mean color and attenuation difference inside the $10 \mathrm{kpc}$ ring excluding the region around $+5 \mathrm{kpc}$ on the near-side of the galaxy along the minor axis because of the presence of the inner spiral arm with enhanced TIR emission and attenuation. In Fig. 15 (left panel), we see that the color profile along the minor axis is systematically redder than along the major axis by on average $\sim 0.5 \mathrm{mag}$. This implies that the attenuation appear to be 
Table 3. Average values and standard deviations (in parenthesis) in $\lg (\mathrm{TIR} / \mathrm{FUV})$, (FUV $-i_{\mathrm{SDSS}}$ ) and $A_{\mathrm{FUV}}$ in each annular region shown in Fig. 12.

\begin{tabular}{ccccccc}
\hline \hline$R(\mathrm{kpc})$ & $\lg (\mathrm{TIR} / \mathrm{FUV})$ & $\left(\mathrm{FUV}-i_{\text {SDSS }}\right)$ & $A_{\mathrm{FUV}}$ & $a$ & $b$ & $N$. Reg. \\
\hline 1. & $2.13(0.11)$ & $7.51(0.07)$ & $1.20(0.14)$ & - & - & 9 \\
3. & $2.15(0.16)$ & $7.18(0.45)$ & $1.31(0.16)$ & $0.38(0.13)$ & $-0.55(0.93)$ & 115 \\
5. & $2.12(0.28)$ & $6.68(0.70)$ & $1.50(0.30)$ & $0.41(0.11)$ & $-0.62(0.74)$ & 381 \\
7. & $2.05(0.23)$ & $6.18(0.75)$ & $1.71(0.40)$ & $0.32(0.10)$ & $0.10(0.64)$ & 590 \\
9. & $2.03(0.26)$ & $5.66(0.71)$ & $2.06(0.44)$ & $0.37(0.15)$ & $-0.05(0.88)$ & 879 \\
11. & $1.91(0.24)$ & $4.96(0.68)$ & $2.45(0.47)$ & $0.42(0.24)$ & $-0.19(1.21)$ & 1200 \\
13. & $1.81(0.26)$ & $4.84(0.69)$ & $2.37(0.47)$ & $0.47(0.27)$ & $-0.45(1.35)$ & 1228 \\
15. & $1.61(0.22)$ & $4.63(0.64)$ & $2.17(0.28)$ & $0.35(0.10)$ & $0.03(0.47)$ & 1380 \\
17. & $1.59(0.21)$ & $4.66(0.80)$ & $2.06(0.33)$ & $0.28(0.09)$ & $0.29(0.42)$ & 615 \\
\hline
\end{tabular}

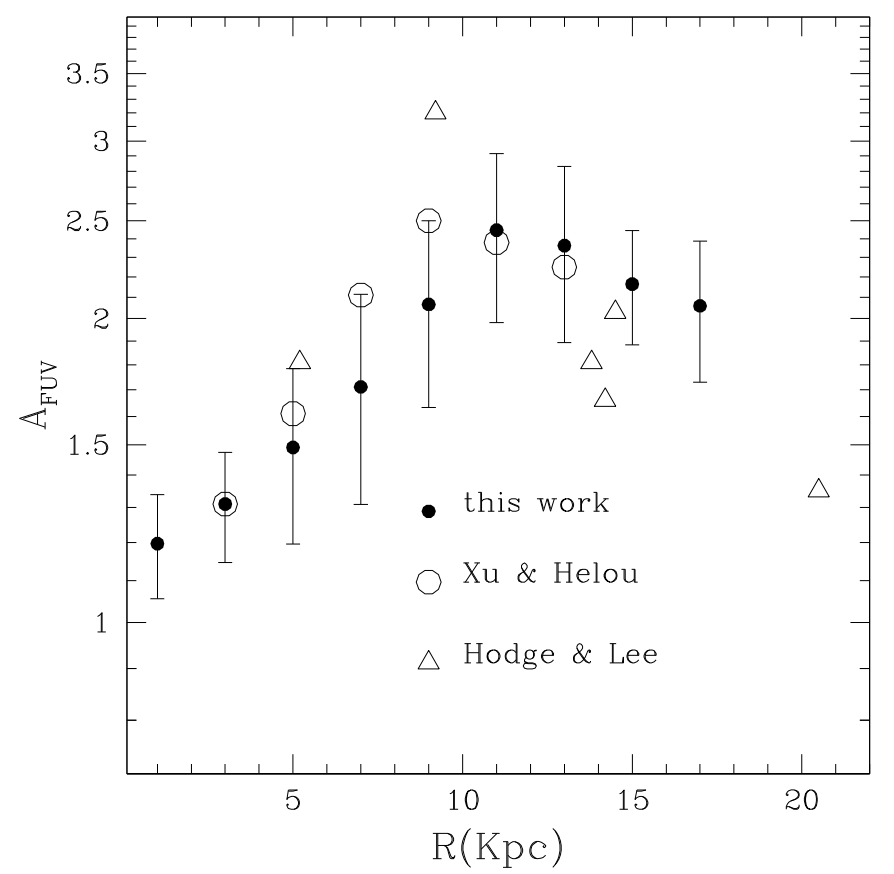

Fig. 13. Average FUV $\left(\lambda_{\text {eff }}=1516 \AA\right)$ attenuations in $2 \mathrm{kpc}$ radial bins from the center of M31 to $\sim 21 \mathrm{kpc}$ obtained by us (filled circles), Xu \& Helou (1996, open circles). Open triangles represent the result of Hodge $\&$ Lee (1988) obtained analyzing 5 fields at different galctocentric distances.

systematically lower on the minor axis by $A_{\mathrm{FUV}} \sim 0.2 \mathrm{mag}$ (Fig. 15 right panel).

Because the major axis also should be affected by this observational bias, the effect should be in principle larger than this. Considering the radial bin at $11 \mathrm{kpc}$ and the one at $3 \mathrm{kpc}$ and considering the color shift toward redder values in the inner bin as being totally due to the bias effect, we estimated the average attenuation at $3 \mathrm{kpc}$ to be higher by $\sim 0.84 \mathrm{mag}$ (Fig. 16) than that derived from the observed colors. This value should be considered as an upper limit because the color shift should be in partially caused by intrinsically redder and older populations than those in the ring, where most of the star formation in M31 is taking place. Nevertheless, even after applying such a significant bias correction, the ring would remain distinctive evident as a maximum of attenuation, as shown in Fig. 16. On the other hand, this exercise highlight the need for a more accurate model to properly account for what happens in the innermost regions.

Xu \& Helou (1996) developed a dust heating/cooling model for M31 based on a radiative transfer code that assumes a sandwich geometry for stars and dust as in Cortese et al. (2008). They found that the mean optical depth $\tau_{\mathrm{v}}$ as viewed from an inclination angle of $77^{\circ}$, increased with radius from $\tau_{v} \sim 0.7$ at $r=2 \mathrm{kpc}$, reaching a peak of $\tau_{\mathrm{v}}=1.6$ near $10 \mathrm{kpc}$, and remaining quite flat out to $14 \mathrm{kpc}$. We used Eqs. (2)-(4) of Cortese et al. (2008) to convert the $\tau_{\mathrm{v}}$ values of Xu \& Helou (1996) into $A_{\mathrm{FuV}}$ magnitudes and obtained $A_{\mathrm{FUV}}=1.23 \mathrm{mag}$ at $2 \mathrm{kpc}$, and $A_{\mathrm{FUV}}=$ 2.50 mag near $10 \mathrm{kpc}$. These results are in good agreement with those shown in Fig. 13 and in Table 3, where we present average values and standard deviations (in parenthesis) in $\lg (\mathrm{TIR} / \mathrm{FUV})$, (FUV $-i_{\text {SDSS }}$ ) and $A_{\mathrm{FUV}}$ in each annular region shown in Fig. 12. The fifth and sixth columns of Table 3 show the linear regression parameters obtained by fitting the data in Fig. 12 (bottom panel) with the relation $\lg (\mathrm{TIR} / \mathrm{FUV})=a\left(\mathrm{FUV}-i_{\text {SDSS }}\right)+b$. The last column shows the number of regions used in each annulus. The radial trend that we derived peaks close to $10 \mathrm{kpc}$ and appears to remain quite flat in the outer regions and to decrease rapidle with decreasing radius in the inner regions. While, considering the uncertainties in the $A_{\mathrm{FUV}}$ estimate, these results are consistent with those of Xu \& Helou (1996), some differences are evident. In the inner side of the ring, our average attenuations tend to be lower (in the range $\sim 0.1 \div 0.5 \mathrm{mag}$ ) with respect to the results of those authors. Although the same sandwich geometry was adopted, the two approaches differ in various aspects. At first, $\mathrm{Xu}$ \& Helou (1996) did not account for the presence of population gradients. While this assumption was motivated by considering that the $(V-R)$ color of M31 appeared rather constant in the disk of M31 (Walterbos \& Kennicutt 1988), the results shown in the previous section, based on a far wider color baseline, indicate that a population gradient is indeed present across the disk of M31. In the absence of population gradients, as discussed in the previous section, one tends to overestimate the attenuation in the region where the age effect is maximal $\left(4<\left(\mathrm{FUV}-i_{\mathrm{SDSS}}\right)<7\right)$, and this is independent of the observational biases discussed previously (which should affect both methods in a similar way). We note that in the inner region at $2 \mathrm{kpc}$, and thus where most of the regions have $\left(\mathrm{FUV}-i_{\mathrm{SDSS}}\right)>7$, the two models appear to be in perfect agreement. On the other hand, the model of Xu \& Helou (1996) solves the radiative transfer problem and fully accounts for the effects of the dust scattering, which are neglected by Cortese et al. (2008). If the scatter was neglected for a fixed observed color, we would have a lower TIR/FUV ratio and would thus predict a lower attenuation. While both of these effects could have contributed to the differences that we see in Fig. 13, the generally good agreement between these results infers that these effects do not dramatically alter our conclusions. As we have shown in the previous sections, old populations dominate the radiation field, so good results can be obtained if the color is fixed to the one of an old population as done in Xu \& Helou (1996). The scattering should affect mostly young stars embedded in the dust, and thus mainly in the spiral-ring structure, but since the radiation field of these stars never dominates the effect should be limited. Finally, we recall that we are 

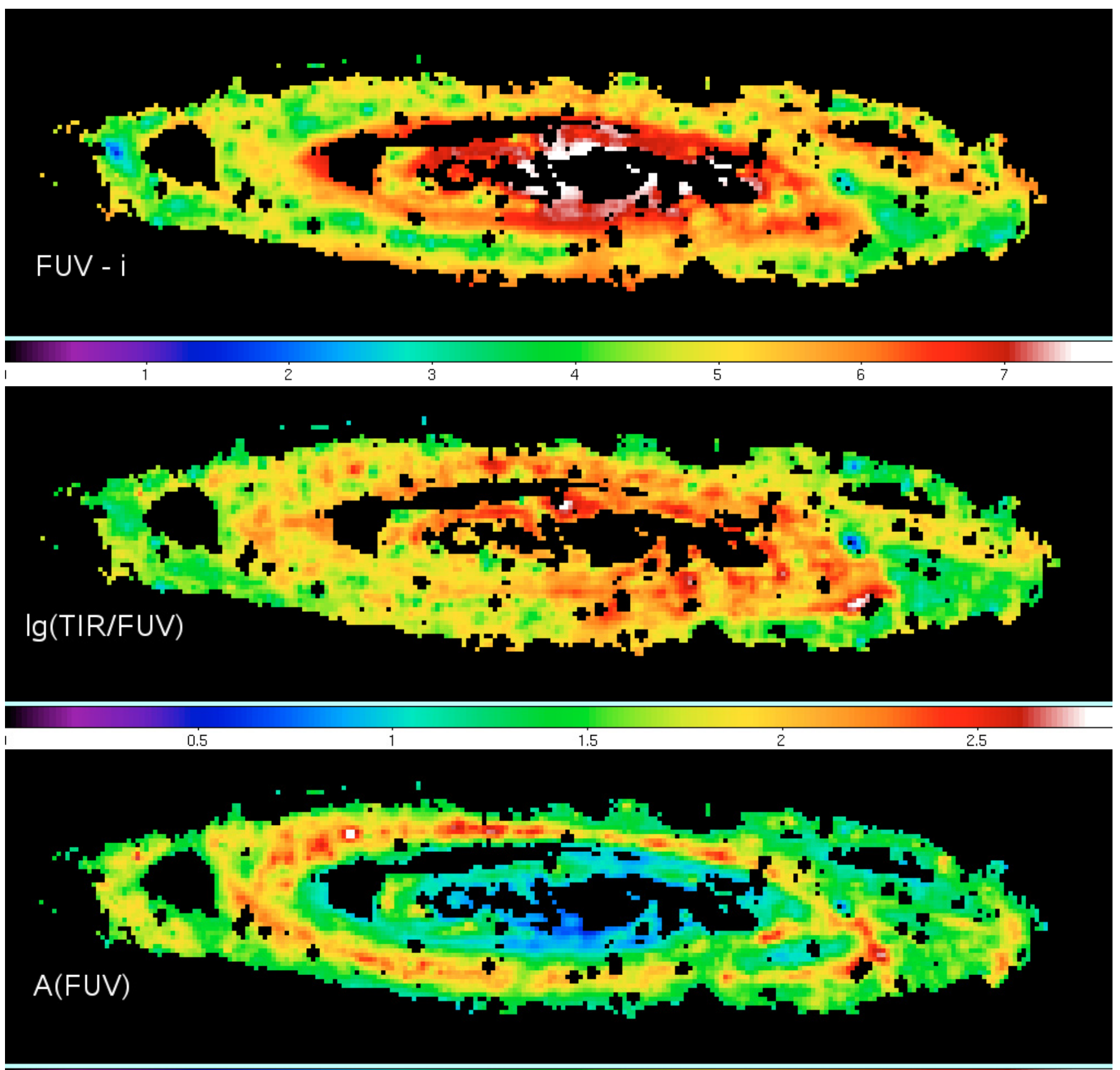

0.5

1.5

2.5

3.5

Fig. 14. From top to bottom: (FUV - $i_{\mathrm{SDSS}}$ ) map, logarithmic TIR/FUV map, and $A_{\mathrm{FUV}}$ attenuation map. Color bars indicate the range of values spanned by the quantities using a linear scale. The first two maps are derived by combining the FUV, $i_{\mathrm{SDSS}}$, and all the infrared maps used in this work. The regions shown here have $S / N>1, \mathrm{FUV} / N U V>0.3$, and $1<F_{8 \mu \mathrm{m}}^{\mathrm{ns}} / F_{24 \mu \mathrm{m}}^{\mathrm{ns}}<10$, and avoid bright sources detected in the $i_{\mathrm{SDSS}}$ band (black dots). The last map shows the attenuations $A_{\mathrm{FuV}}$ derived using the recipes of Cortese et al. (2008) and the two above maps. The attenuation is maximum in the $10 \mathrm{kpc}$ ring, where it reaches a mean value of $2.5 \pm 0.7 \mathrm{mag}$, and declines more strongly towards the inner regions of the galaxy than towards the outer regions. These maps are available in electronic form at the CDS via anonymous ftp to cdsarc.u-strasbg.fr (130.79.128.5) or via http://cdsweb.u-strasbg.fr/cgi-bin/qcat?]/A+A/507/283.

comparing results obtained at different spatial resolutions $\left(40^{\prime \prime} / \mathrm{px}\right.$ in our case, and $2^{\prime} / \mathrm{px}$ in the case of Xu \& Helou 1996) and that the accuracy of Spitzer, GALEX, and SDSS maps is certainly superior to the measurements used by Xu \& Helou (1996). In particular, using IRAS images the cold dust emission and thus the TIR emission should be less accurately constrained than using Spitzer data, since the longest wavelength measurement of IRAS is at $100 \mu \mathrm{m}$, whereas for Spitzer it is at $160 \mu \mathrm{m}$ close to the FIR spectrum peak of M31 (Fig. 3). In Fig. 13, we also show the comparison of our results with those of Hodge \& Lee (1988), who used two color diagrams to determine the average reddenings of 5 fields at different M31 galactocentric distances. We converted the reddening values of Hodge \& Lee (1988) to the optical depths along the line of sight $\left(\tau_{\mathrm{v}}\right)$ by multiplying by a factor of $2 \times 0.921 \times 2.8$ as in Xu \& Helou (1996), then we proceeded as before to convert the $\tau_{\mathrm{v}}$ in $A_{\mathrm{FUV}}$. Although the radial trend always peaks at $10 \mathrm{kpc}$, a larger scatter with respect to the results of $\mathrm{Xu} \&$ Helou (1996) is visible. This may be caused 

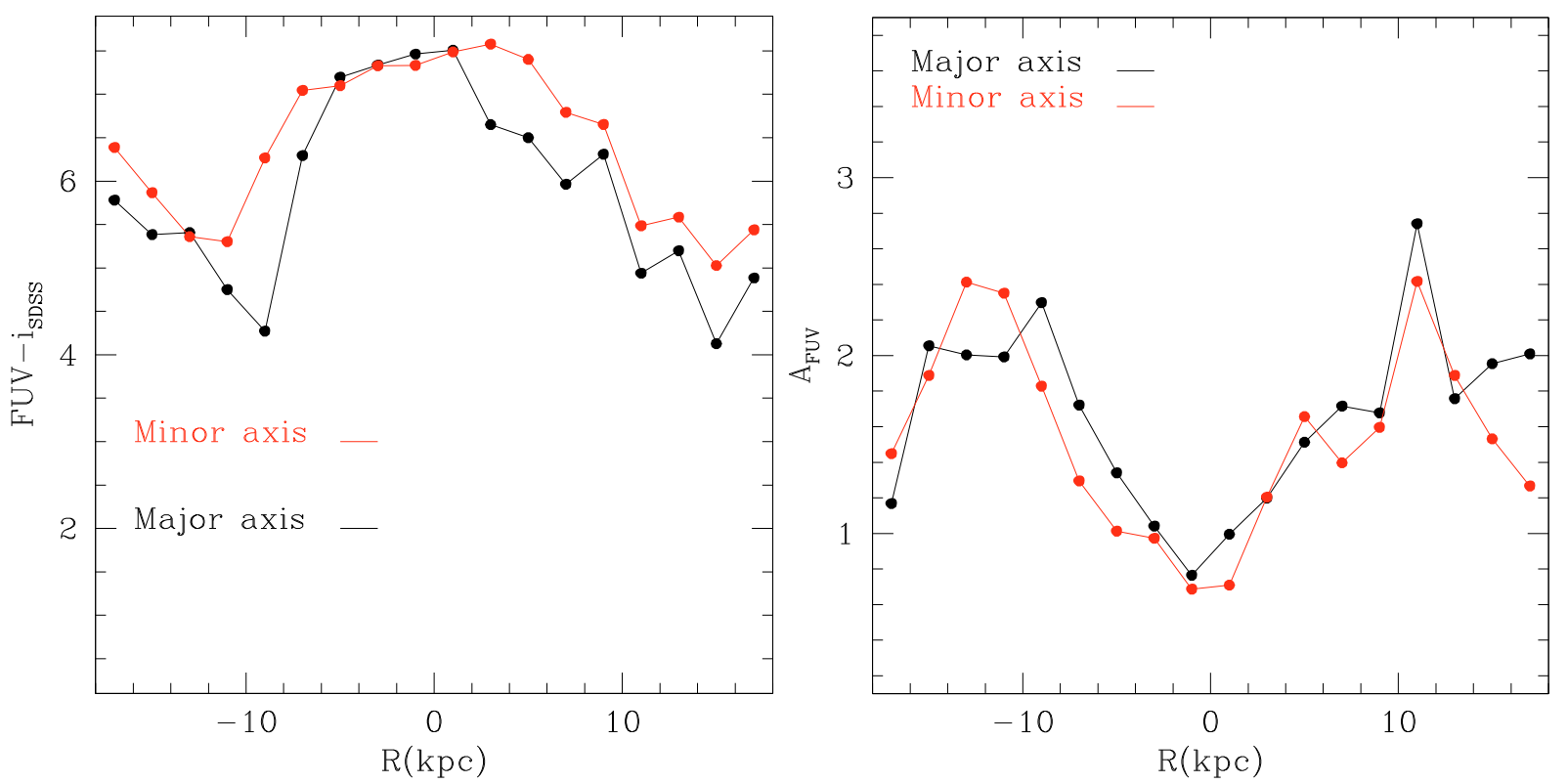

Fig. 15. Left panel: (FUV - $i_{\mathrm{SDSS}}$ ) color profiles along the minor (red line) and major axis (black line). Right panel: average attenuations along the minor and major axis. The profiles are derived by considering regions with $S / N>1$. Each point is the average value of the colors (attenuations) inside a $200^{\prime \prime}$-wide $(0.75 \mathrm{kpc})$ stripe, centered on the major and minor-axis corresponding to each of the $2 \mathrm{kpc}$ annular regions shown in Fig. 2.

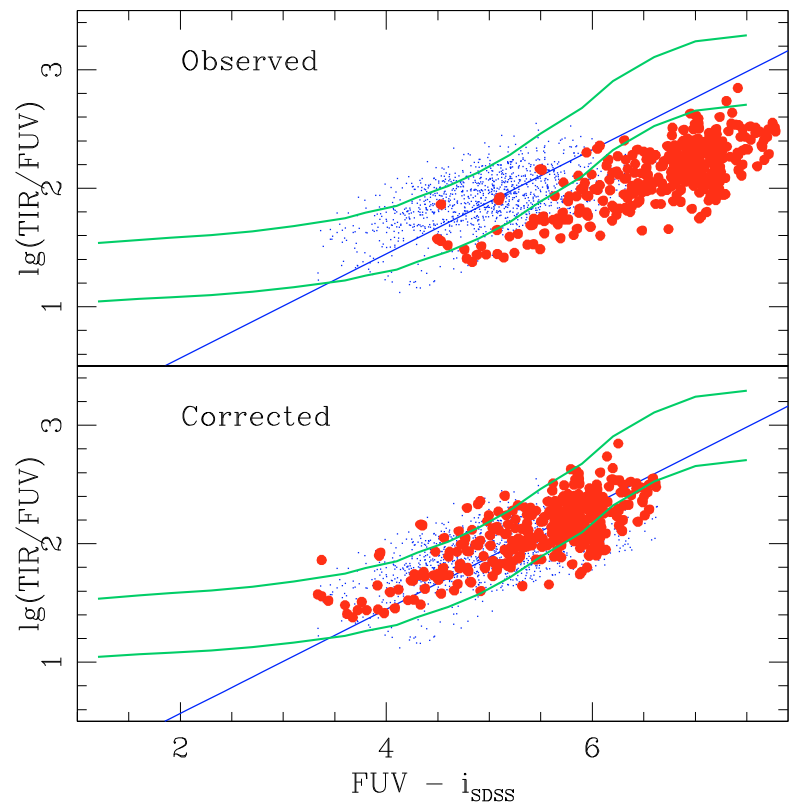

Fig. 16. Upper panel: observed colors and TIR/FUV ratios for regions in the $3 \mathrm{kpc}$ radial bin (big red points) and in the $11 \mathrm{kpc}$ radial bin (small blue points). Lower panel: same as upper panel, but the observed colors in the inner radial bin are shifted by the average color difference with respect to the best-fit linear model in the outer ring (blue line).

by both ourselves and Xu \& Helou (1996) analyzing radial averages, whereas the results of Hodge \& Lee (1988) are based on 5 selected regions.

The $10 \mathrm{kpc}$ ring is the locus where gas density and star formation activity also reach their maximum values across the disk of M31 (e.g., Braun et al. 2009; Nieten et al. 2006; Reddish 1962; Hodge 1979; Hodge \& Lee 1988), thus it is unsurprising that the dust density also peaks at the ring as found in previous studies.
Another limitation of the approach that we followed consists of assuming that each one of the cells that we analyzed is independent of the others, and thus the radiation field of each region is resposible only for the dust heating in that region. As the mean free path of photons is typically larger than the dimension of the regions analyzed here $\left(40^{\prime \prime} / \mathrm{px}, \sim 670 \mathrm{pc} / \mathrm{px}\right)$, it is possible that photons coming from nearby bright regions could contribute significantly to dust heating. Nevertheless, this effect is likely to have a limited impact on our conclusions. At first, because dust heated by stars in star-forming regions is not the dominant source of emission in the FIR in M31, as indicated by the hot dust component emission at $24 \mu \mathrm{m}$ being overall $<7 \%$ of the TIR emission, as shown in Sect. 3.1. Secondly, the smooth distribution of the M31 surface brightness in the optical $(\lambda>4000 \AA$ from where most of the dust-heating radiation comes) is indicative of homogeneous conditions among adjacent regions for which a local equilibrium between photons transmitted and received in closeby regions can be assumed.

Finally, using the correlation between the $24 \mu \mathrm{m}$ and the TIR emission shown in Fig. 6, we derived the reddening map of M31 at $6 " / p x$ (Fig. 17). We then applied the reddening correction to the observed SDSS map (Fig. 18). The most prominent dust lanes and absorption features are clearly consistent with the reddening map, and the ring emission at $10 \mathrm{kpc}$ appears to be very significant. We note that in Figs. 17, and 18 we show the whole maps for clarity, but regions where optical, UV, and infrared point-like sources are visible should be interpreted with care. We discussed the issue of contaminants and how we corrected for them in Sect. 2.

\section{Conclusions}

In this paper, we have studied the dust properties of M31. The major results that we reached are the following: (i) from the study of the infrared spectrum of M31, we have found that the mean intensity of the radiation field heating the dust is globally low (typically $U<2$ ); (ii) the dust mass $\left(M_{\text {dust }}\right)$ estimate remains uncertain because of a lack of submillimeter 


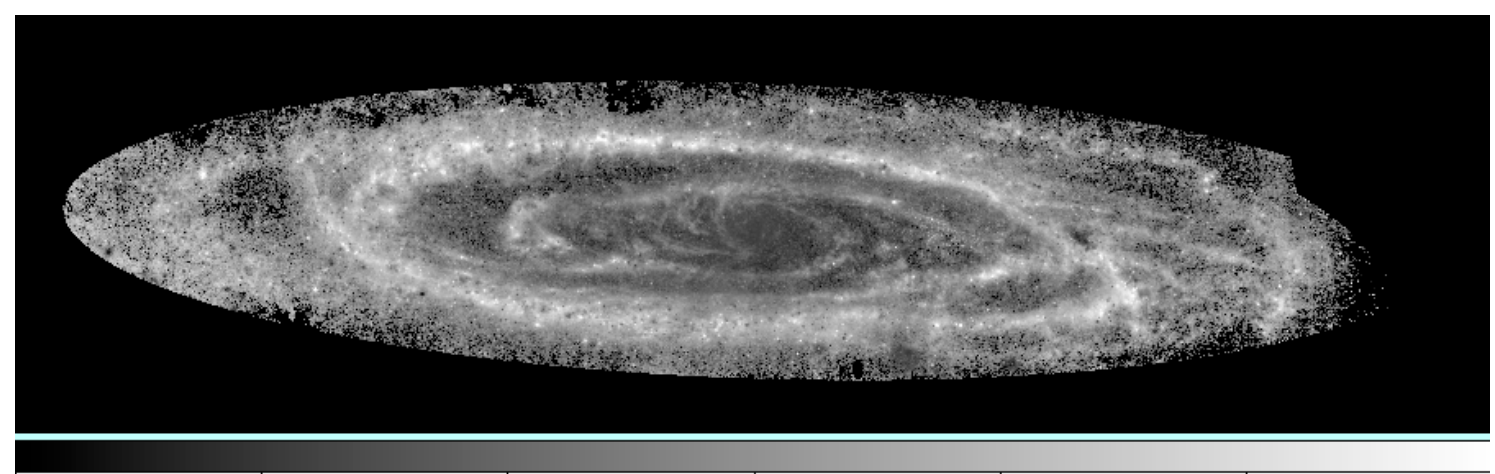

0.1

0.2

0.3

0.5

Fig. 17. $E(B-V)$ map at $6 "$ px resolution $(\sim 100 \mathrm{pc} / \mathrm{px}$ along the plane of M31). This map is available in electronic form at the CDS via anonymous ftp to cdsarc.u-strasbg.fr (130.79.128.5) or via http://cdsweb.u-strasbg.fr/cgi-bin/qcat? J/A+A/507/283
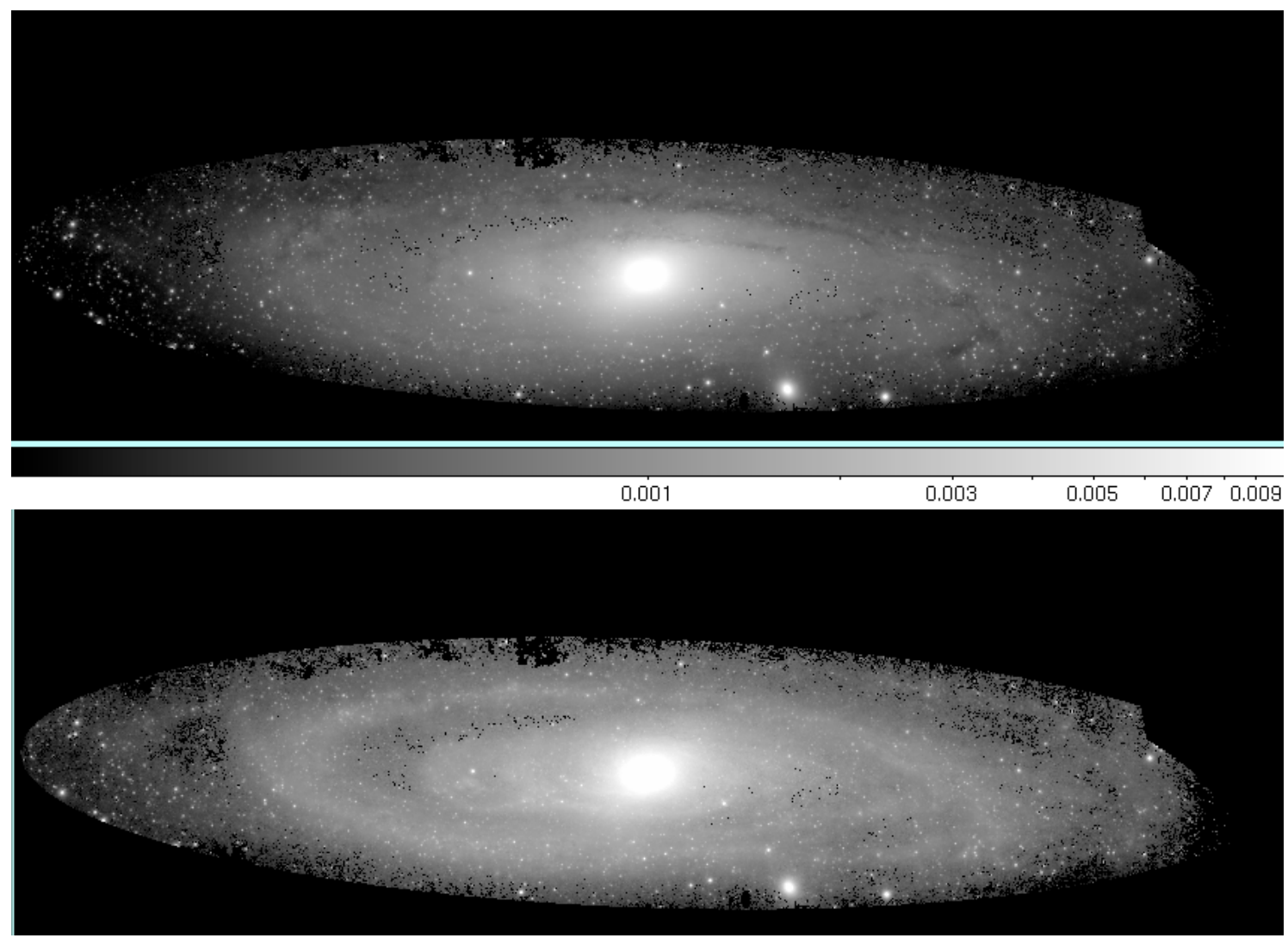

Fig. 18. Upper panel: original SDSS image in the $i$-band. Lower panel: SDSS $i$-band image dereddened with our extinction map. For clarity, all regions are shown here. Regions with bright point-like sources and/or occasional negative fluxes in some (UV/Optical/IR) maps (black regions) are unreliable. The colorbar shows intensity levels in Jansky in logarithmic units. Images have 6"/px resolution.

observations, but we have found that $M_{\text {dust }} \gtrsim 1.1 \times 10^{7} M_{\odot}$, the value given by the best-fit model being $7.6 \times 10^{7} M_{\odot}$ in good agreement with that inferred from $\mathrm{CO}$ and $\mathrm{HI}$ observations; (iii) the abundance of polyciclic aromatic hydrocarbon (PAH) particles in M31 is high ( $\left.>3 \% M_{\text {dust }}\right)$ across the spiral-ring structure of M31; (iv) we demonstrated the existence of a correlation between the observed TIR/FUV emission ratios and the color $\left(\mathrm{FUV}-i_{\text {SDSS }}\right.$ ) over the entire spiral-ring structure of M31; (v) this correlation is not in agreement with the $I R X-\beta$ relationship of starburst galaxies, thus color changes are in general not driven by dust attenuations; (vi) we found that according to the prescription of models that consider age-dependent dust heating, the observed correlation could be evidence of a population gradient and a quite homogeneous attenuation of the analyzed regions; (vii) in $83 \%$ of the regions between $8 \mathrm{kpc}<r<12 \mathrm{kpc}$, the dust absorbs more than $50 \%$ of the energy at $\lambda>4000 \AA$ and it appears to be mainly heated by populations a few Gyr old, which could provide a good interpretation of the low mean intensity of the radiation field that we found from the independent analysis of the infrared spectrum; (viii) we found that the mean attenuation in $2 \mathrm{kpc}$-wide radial bins reaches the maximum value near $10 \mathrm{kpc}$ and decreases more rapidly with radius in the inner than the outer regions of the galaxy, and that regions with higher TIR emission also have enhanced attenuations; (ix) we finally produced an attenuation map of M31 at $6 " /$ px resolution ( 100/px along the plane of M31).

Future work will investigate in more detail the dependence of the dust attenuation values on other parameters (e.g., color, 
metallicity), the star formation rate, and the modeling of the stellar populations in M31.

The maps presented in Fig. 14 and the E(B-V) map of Fig. 17 are available via CDS.

Acknowledgements. We acknowledge the useful comments of the anonymous referee. This research was supported by the DFG cluster of excellence "Origin and Structure of the Universe" (www . universe-cluster . de).

\section{References}

Barmby, P., Huchra, J. P., Brodie, J. P., et al. 2000, AJ, 119, 727 Barmby, P., Ashby, M. L. N., Bianchi, L., et al. 2006, ApJ, 650, 45 Bendo, G. J., Draine, B. T., Engelbracht, C. W., et al. 2008, MNRAS, 389, 629 Bianchi, L., Clayton, G. C., Bohlin, R. C., et al. 1996, ApJ, 471, 203 Bigiel, F., Leroy, A., Walter, F., et al. 2008, AJ, 136, 2846 Buat, V. 1992, A\&A, 264, 444

Buat, V., Iglesias-Páramo, J., Seibert, M., et al. 2005, ApJ, 619, L51

Braun, R., et al. 2009 [arXiv: 0901.4154B]

Bruzual, G., \& Charlot, S. 2003, MNRAS, 344, 1000

Calzetti, D., Kennicutt, R. C., Engelbracht, C. W., et al. 2007, ApJ, 666, 870

Charlot, S., \& Fall, S. M. 2000, ApJ, 539, 718

Cortese, L., Boselli, A., Franzetti, P., et al. 2008, MNRAS, 386, 1157

Draine, B. T., \& Li, A. 2007, ApJ, 657, 810

Draine, B. T., Dale, D. A., Bendo, G. D., et al. 2007, ApJ, 663, 866D Gil de Paz, A., Boissier, S., Madore, B. F., et al. 2007, ApJS, 173, 185 Fan, Z., Ma, J., de Grijs, R., et al. 2008, MNRAS, 385, 1973

Gavazzi, G., Bonfanti, C., Sanvito, G., et al. 2002, ApJ, 576, 135

Gordon, K. D., Clayton, G. C., Witt, A. N., et al. 2000, ApJ, 533, 236

Gordon, K. D., Bailin, J., Engelbracht, C. W., et al. 2006, ApJ, 638, 87
Gordon, K. D., Engelbracht, C. W., Rieke, G. H., et al. 2008, ApJ, 682, 336 Haas, M., Lemke, D., Stickel, M., et al. 1998, A\&A, 338, 33

Helou, G., Roussel, H., Appleton, P., et al. 2004, ApJS, 154, 253

Hodge, P. W. 1979, AJ, 84, 744

Hodge, P. 1980, AJ, 85, 376

Hodge, P. W., \& Kennicutt, R. C. 1982, AJ, 87, 264

Hodge, P. W., \& Lee M. G. 1988, ApJ, 329, 651

Inoue, A. K. 2005, MNRAS, 359, 171

Inoue, A. K., Buat, V., Burgarella, D., et al. 2006, MNRAS, 370, 380

Kennicutt, R. C., Jr., Calzetti, D., Walter, F., et al. 2007, ApJ, 671, 333

Kong, X., Charlot, S., Brinchmann, J., et al. 2004, MNRAS, 349, 769

Leroy, A. K., Walter, F., Brinks, E., et al. 2008, AJ, 136, 2782

Kraemer, K. E., Price, S. D., Mizuno, D. R., et al. 2002, AJ, 124, 2990

Li, A., \& Draine, B. T. 2001, ApJ, 554, 778

Mathis, J. S., Mezger, P. G., \& Panagia, N. 1983, A\&A, 128, 212

Madden, S. C., Galliano, F., Jones, A. P., et al. 2006, A\&A, 446, 877

Meurer, G. R., Heckman, T. M., Leitherer, C., et al. 1995, AJ, 110, 2665

Meurer, G. R., Heckmann, T. M., \& Calzetti, D. 1999, ApJ, 521, 64

Nieten, Ch., Neininger, N., \& GuÃl'lin, M. 2006, A\&A, 453, 459

Odenwald, S., Newmark, J., \& Smoot, G. 1998, ApJ, 500, 554

Rice, W., Lonsdale, C. J., Soifer, B. T., et al. 1988, ApJS, 68, 91

Reddish, V. C. 1962, Zs. Ap., 56, 194

Salim, S., Rich, R. M., Charlot, S., et al. 2007, ApJS, 173, 267

Schlegel, D. J., Finkbeiner, D. P., \& Davis, M., ApJ, 500, 525

Schmidtobreick, L., Haas, M., \& Lemke, D. 2000, A\&A, 363, 91

Smith, J. D. T., Draine, B. T., Dale, D. A., et al. 2007, ApJ, 656, 770

Unwin, S. C. 1983, MNRAS, 205, 787

Weingartner, J. C., \& Draine, B. T. 2001, ApJ, 548, 296

Walterbos, R. A. M., \& Kennicutt, R. C. 1988, A\&A, 198, 61

Xu, C., \& Helou, G. 1996, ApJ, 456, 163

York, D. G., Adelman, J., Anderson, J. E., et al. 2000, AJ, 120, 1579 